\title{
STRICHARTZ ESTIMATES FOR A SCHRÖDINGER OPERATOR WITH NONSMOOTH COEFFICIENTS
}

\author{
GIGLIOLA STAFFILANI AND DANIEL TATARU
}

\begin{abstract}
We prove Strichartz type estimates for the Schrödinger equation corresponding to a second order elliptic operator with variable coefficients. We assume that the coefficients are a $C^{2}$ compactly supported perturbation of the identity, satisfying a nontrapping condition.
\end{abstract}

\section{INTRODUCTION}

The Strichartz estimates provide a good quantitative measure of the dispersion phenomena for various dispersive equations such as wave, Schrödinger and KdV. As it turns out, they are very useful in the study of various corresponding semilinear equations. A new difficulty arises in the study quasilinear problems, namely that one needs to deal with operators with rough coefficients.

Recently there has been considerable progress in deriving Strichartz type results for the linear wave equations with nonsmooth coefficients. As it turns out, in order to obtain full dispersive estimates one essentially needs to control two derivatives of the coefficients. Wave operators with $C^{2}$ coefficients are considered in Smith [15] in $2+1$ and $3+1$ dimensions and in Tataru [20] in any dimension; this can be further relaxed to $\partial^{2} g \in L^{1}\left(L^{\infty}\right)$, see Tataru [18]. As the work of Chemin and Bahouri [2],[1], Tataru [19],[18] and finally Klainerman and Rodnianski [13] has showed, these results are fundamental in order to improve existence and uniqueness results for quasilinear wave equations. In this paper we derive Strichartz type estimates for a Schrödinger operator with nonsmooth coefficients. The situation here is more complex because a Schrödinger operator does not enjoy the property of finite speed of propagation. A localization argument, trivial in the case of the wave operator, here requires considerably more care. But once this localization has been made rigorous, the setting becomes very much like the one used in Tataru [20] and the results follow by applying the method of the FBI transform. For the constant coefficient case we refer the reader to the survey article [6] and also to the endpoint result in [10].

In this first paper, in order to make the localization simpler we assume that outside a large ball the operator we consider has constant coefficients. We plan to investigate the same questions in a more general setting in a future paper.

Before we go to the details of the presentation of the problem we considerin this paper, we would like to recall that the Scrödinger operator, with smooth variable coefficients, appears in many papers concerning questions on well-posedness and smoothing effect (see Doi [5] [4], Creg, Kappler and Strauss [3], Kapitanski and

Date: December 26, 2007.

The first author is partially supported by NSF grant DMS-9800879 and by the Terman Award. The second author is partially supported by NSF grant DMS-9622942 . 
Safanov [9] [8], Kenig, Ponce and Vega [11] and Rolvung [14]). As mentioned above, in our work the coefficients are instead relatively rough. But the FBI approach is very effective in treating this nonsmooth situation.

We now start by introducing the initial value problem (IVP) we will be working with and some notations. We write the IVP with a Schrödinger operator of variable coefficients as

$$
\left\{\begin{array}{l}
\left(i \partial_{t}-\sum_{k, j=1}^{n} \partial_{k} a^{k j}(t, x) \partial_{j}\right) u=0 \\
u(x, 0)=u_{0}(x), \quad x \in \mathbb{R}^{n}
\end{array}\right.
$$

where

- (A1)

$a^{k j} \in\left[L^{\infty}\left(C^{1,1}\right) \cap C^{0,1}\left(L^{\infty}\right)\right]\left(\mathbb{R} \times \mathbb{R}^{n}\right), \quad a^{k j}(t, x)=\delta^{k j}$ outside $\mathbb{R} \times B(0,1)$.

- (A2)

$a^{k j}(t, x)$ is real and symmetric and there exists $\delta>0$ such that

$$
\delta|\xi|^{2} \leq \sum_{k, j=1}^{n} a^{k j}(t, x) \xi_{k} \xi_{j} \leq \delta^{-1}|\xi|^{2},
$$

for any $t \in \mathbb{R}, x, \xi \in \mathbb{R}^{n}$.

In the rest of the paper we denote with $P$ the differential operator

$$
P=i \partial_{t}-\sum_{k, j=1}^{n} \partial_{k} a^{k j}(x) \partial_{j}
$$

and

$$
\Delta_{a}=\sum_{k, j=1}^{n} \partial_{k} a^{k j}(x) \partial_{j}
$$

We write $a(x, \xi)=\sum_{k, j=1}^{n} a^{k j}(x) \xi_{k} \xi_{j}$ and note that by (A1) and (A2) we have $a(x, \xi)>0$. We also denote with $H_{q}$ the Hamiltonian vector field associated to a function $q \in C^{1}\left(T^{*} \mathbb{R}^{n}\right)$, that is

$$
H_{q}=\sum_{j=1}^{n}\left(\partial_{\xi_{j}} q \partial_{x_{j}}-\partial_{x_{j}} q \partial_{\xi_{j}}\right) .
$$

It is not difficult to prove that, by (A2), $H_{a}$ is complete in $T^{*} \mathbb{R}^{n}$. We denote with $S_{\text {trap }}$ the set of all $(x, \xi) \in S^{*} \mathbb{R}^{n}=\{(x, \xi) ; a(x, \xi)=1\}$ such that the complete integrable curve of $H_{a}$ through $(x, \xi)$ is contained in a compact set. Then property (A3) is defined as

- (A3) $S_{\text {trap }}=\emptyset$.

Remark 1. One could also consider an operator with lower order terms

$$
\left.Q=\left(i \partial_{t}-\sum_{k, j=1}^{n} \partial_{k} a^{k j}(t, x) \partial_{j}\right)+\sum_{j=1}^{n} b^{j}(t, x) \partial_{j}+c(t, x)\right)
$$

Here we decided to consider only the case $b(x, t)=c(x, t)=0$ in order to simplify the presentation. However, our arguments apply with few changes if for instance $b, c$ are bounded and compactly supported.

We now state the main theorem of the paper. 
Theorem 1. Assume (A1), (A2) and (A3), then for any $T>0$, the unique solution $u$ of (1) satisfies

$$
\|u\|_{L^{q}\left([0, T], L^{r}\right)} \leq C_{T}\left\|u_{0}\right\|_{L^{2}}
$$

for any pair $^{1}(q, r)$ such that

$$
\begin{array}{lll}
\frac{2}{q}+\frac{n}{r}=\frac{n}{2}, & 2 \leq q \leq \infty & \text { for } n \neq 2 \\
\frac{2}{q}+\frac{2}{r}=1, & 2<q \leq \infty, & \text { for } n=2 .
\end{array}
$$

Remark 2. Using arguments similar to those in [18] one can relax the $C^{2}$ assumption on the coefficients to

$$
\partial_{x}^{2} a^{j k}, \partial_{t} a^{j k} \in L_{x_{j}}^{1}\left(L_{t, x_{j}^{\prime}}^{\infty}\right), \quad j=1, \cdots, n
$$

where $x_{j}^{\prime}$ denotes the set of coordinates complementary to $x_{j}$. This suffices in order to guarantee the same regularity of the Hamilton flow which we use in this paper.

Note that for $(q, r)=(\infty, 2)(6)$ is a straightforward energy estimate, therefore by interpolation it suffices to prove it for $q<r$. The proof of Theorem 1 relies on the following localization. We split the solution $u$ of problem (1) into $v=\chi u$ and $w=(1-\chi) u$, where $\chi$ is a smooth characteristic function of the set $B(0,1)$ in $\mathbb{R}^{n}$. Observe that supp $w \subset \mathbb{R}^{n} / B_{(0,1)}$, where $\Delta_{a}=\Delta$. Then the functions $v, w$ solve the inhomogeneous Cauchy problems

$$
\left\{\begin{array}{l}
\left(i \partial_{t}-\Delta_{a}\right) v=f(x, t) \\
v(x, 0)=\chi(x) u_{0}(x)
\end{array}\right.
$$

respectively

$$
\left\{\begin{array}{l}
\left(i \partial_{t}-\Delta\right) w=-f(x, t) \\
w(x, 0)=(1-\chi(x)) u_{0}(x)
\end{array}\right.
$$

The inhomogeneous term $f$ has compact spatial support and is given by

$$
f=[P, \chi] u \text {. }
$$

Our first step, in the next section, is to use local smoothing to establish the sharp $L^{2}$ regularity of $v$ and $f$. Using an argument ${ }^{2}$ due to Doi $[5,4]$ we prove that (see Theorem 2)

$$
\|v\|_{L_{t}^{2} H_{x}^{1 / 2}}+\|f\|_{L_{t}^{2} H_{x}^{-1 / 2}} \lesssim\left\|u_{0}\right\|_{L_{x}^{2}} .
$$

In Section 3 we establish the $L^{q}\left(L^{r}\right)$ estimates for $w$,

$$
\|w\|_{L_{t}^{q} L_{x}^{r}} \lesssim\|f\|_{L_{t}^{2} H_{x}^{-1 / 2}}+\|w(0)\|_{L_{x}^{2}} .
$$

For this we use a classical approach. Note, however, that here the dispersive estimates are combined with local smoothing, therefore we cannot employ directly the dispersive estimates for the constant coefficient Schrödinger equation. It is essential that the inhomogeneous term $f$ has compact spatial support.

\footnotetext{
${ }^{1}$ Such a pair is usually called admissible.

${ }^{2}$ In Doi's paper the coefficients $a^{k j}$ are independent of $t$, but as he observes in [4], the proofs work also when the coefficients are functions also of the time variable.
} 
The last part of the paper is devoted to the proof of the Strichartz estimate for the ( compactly supported ) function $v$, namely

$$
\|v\|_{L^{q}\left([0, T], L^{r}\right)} \leq\|v\|_{L_{T}^{2} H_{x}^{1 / 2}}+\|P v\|_{L_{T}^{2} H_{x}^{-1 / 2}} .
$$

This inequality is obtained using arguments similar to those in Tataru [20]. The main idea is to use the FBI transform to construct a microlocal parametrix for the Schrödinger equation. The parametrix is good enough so that it yields the Strichartz estimates by using stationary phase methods.

\section{LOCAL SMOOTHING}

We first introduce a new space of functions that appears in Doi $[5,4]$.

Definition 1. Let $\lambda: \mathbb{R}^{+} \longrightarrow \mathbb{R}^{+}$be a positive integrable smooth nonincreasing function. We define

$$
X_{\lambda}^{s}=\left\{f(x) /\|f\|_{X_{\lambda}^{s}}<\infty\right\}
$$

where

$$
\|f\|_{X_{\lambda}^{s}}^{2}=\left(\lambda(|x|) D^{s+1 / 2} f, D^{s+1 / 2} f\right)+\|f\|_{H^{s}}^{2},
$$

where $(\cdot, \cdot)$ is the inner product in $L\left(\mathbb{R}^{n}\right)$.

The local smoothing result we need is contained in the following well-posedness theorem:

Theorem 2. Assume (A1), (A2) and (A3). Then there exists a unique solution $u$ of (1) such that $u \in C\left([0, T], L^{2}\right) \cup L^{2}\left([0, T], X_{\lambda}^{0}\right)$. Moreover

$$
\|u\|_{L^{2}\left([0, T], X_{\lambda}^{0}\right)} \lesssim\left\|u_{0}\right\|_{L^{2}}
$$

Regardless of the choice of the function $\lambda$ this implies the estimate (9).

Remark 3. The proof of this theorem is due to Doi $[5,4]$ in the case when the coefficients $a^{k j}$ are smooth and asymptotically flat. Then one obtains the estimate

$$
\|u\|_{L^{2}\left([0, T], X_{\lambda}^{s}\right)} \lesssim\left\|u_{0}\right\|_{H^{s}}
$$

for any $s \in \mathbb{R}$. But if one wants it only for $s=0$ ( $L^{2}$ - theory), then the weaker assumption $a^{k j} \in C^{2}$ is enough.

For completeness we recall below the two propositions which, combined with a classical Garding inequality, give Theorem 2.

Proposition 1. Assume (A1) and (A2) and that there exists $q \in S^{0}$ such that

$$
H_{a} q \geq-1 \text {. }
$$

Then there exists a unique solution $u$ of (1) such that

$$
u \in C\left([0, T], L^{2}\right) \cup L^{2}([0, T], \tilde{X}),
$$

where $^{3}$

$$
\|u\|_{\tilde{X}}^{2}=\left(\left(H_{a} q\right)^{w}(x, D) u, u\right)+C\|u\|_{L^{2}}^{2} .
$$

${ }^{3}$ In the standard Weyl calculus one writes

$$
\left(q^{w}(x, D) u\right)(x)=(2 \pi)^{-n} \iint e^{i(x-y) \cdot \xi} q((x+y) / 2, \xi) u(y) d y d \xi,
$$

(see for example [7], Chapter 18). 
with a large constant $C$. Moreover,

$$
\int_{0}^{T}\|u\|_{\tilde{X}}^{2}(\tau) d \tau \lesssim\left\|u_{0}\right\|^{2} .
$$

Proposition 2. Assume (A1), (A2) and (A3). Let $\lambda: \mathbb{R}^{+} \longrightarrow \mathbb{R}^{+}$be an integrable nonincreasing positive smooth function. Then there exists $q \in S^{0}$ and $c>0$ such that

$$
H_{a} q \geq c \lambda(|x|)|\xi|-1 \text { in } T^{*} \mathbb{R}^{n}
$$

Remark 4. The proof of the propositions is basically due to Doi [5, 4]. It is not difficult to see how they imply Theorem 2. One considers the $L^{2}$ energy functional

$$
E(u(t))=<Q^{w} u(t), u(t)>_{L^{2}}+C\|u(t)\|_{L^{2}} .
$$

Then

$$
\frac{d}{d t} E(u(t))=<i\left[A, Q^{w}\right] u, u>_{L^{2}} .
$$

But modulo an $L^{2}$ bounded error, $i\left[A, Q^{w}\right] \approx\left(H_{a} q\right)^{w}$. Now the conclusion of the Theorem follows from Garding's inequality combined with Gronwall's inequality.

The main difference here is that we are not dealing with classical symbols, but with nonsmooth symbols of the type described in Taylor [21] III, p. 45. To convince the reader that the assumptions on the symbols are sufficient we state and prove below, under our conditions, the main lemma used in Doi's argument. The function $q$ in Proposition 2 can be obtained after an algebraic transformation from the function $q$ below.

Lemma 1. Assume (A1), (A2) and (A3). Then there exists a 0-homogeneous symbol $q: \mathbb{R} \times T^{*} \mathbb{R}^{n} \longrightarrow \mathbb{R}$ such that

$$
\begin{aligned}
\left|\partial_{t}^{m} \partial_{\xi}^{\alpha} \partial_{x}^{\beta} q\right| & \leq C_{\alpha \beta m}(1+|x|)|\xi|^{-|\alpha|}, \\
H_{a} q & \gtrsim|\xi|
\end{aligned}
$$

for any $\xi, x \in \mathbb{R}^{n}$ and any multiindex $(m, \alpha, \beta)$.

Proof. We first observe that it suffices to do this for fixed $t$. Indeed, if a function $q$ satisfies the above conditions for some $t$ then it satisfies the same conditions for $t$ nearby. Hence we can use a partition of unit with respect to the time variable to put together the choices of $q$ for fixed time.

Recall that $a^{k j}(x)=\delta^{k j}$ for any $x \in \mathbb{R}^{n} / B_{1}$. Then we define

$$
q_{1}(x, \xi)=x \cdot \xi|\xi|^{-1}
$$

and we notice that for $x \in \mathbb{R}^{n} / B_{1}$

$$
q_{1}(x, \xi)=x \cdot \xi a^{-\frac{1}{2}}
$$

and

$$
H_{a} q_{1}=\sum_{j} 2 \xi_{j} \partial_{x_{j}} q_{1}=|\xi| .
$$

We have to modify $q_{1}$ smoothly inside the ball so that (14) holds everywhere. Set $b=\sqrt{a}$ and let $\Phi_{h}$ be the $H_{b}$ flow and $\Pi$ the natural projection from $T^{*} \mathbb{R}^{n}$ into 
$\mathbb{R}^{n}$. Let $\chi \in C_{0}^{\infty}$ be a positive function such that $\chi=1$ on $|x| \leq 2$ and $\chi=0$ on $|x| \geq 4$. We set $\chi_{r}(x)=\chi(x / r)$, for $r>0$. We define

$$
q_{2}(x, \xi)=-\int_{0}^{\infty} \chi_{2}\left(\Pi \circ \Phi_{h}(x, \xi)\right) d h,
$$

for any $(x, \xi) \in\left\{(x, \xi) \in T^{*} \mathbb{R}^{n} a(t, x, \xi)=1\right\}=S^{*} \mathbb{R}^{n}$. Then $H_{b} q_{2}=\chi_{2}$ and $q_{2}$ is bounded by the non-trapping condition (A3). Since the flow $H_{b}$ is of class $C^{1}$, it also follows that $q_{2} \in C_{l o c}^{1}\left(S^{*} \mathbb{R}^{n}\right)$. We claim that the function

$$
q(x, \xi)=q_{1}(x, \xi)+M^{1 / 2} \chi_{M}(x) q_{2}(x, \xi),
$$

satisfies

$$
H_{a} q \gtrsim 1 \quad \text { in } S^{*} \mathbb{R}^{n}
$$

provided that $M$ is large enough. Indeed, for $M>4$ we have

$$
H_{a} q=H_{a} q_{1}+M^{1 / 2} \chi_{2}(x)+M^{-\frac{1}{2}} a_{\xi} \chi_{x}\left(\frac{x}{M}\right) .
$$

If $|x|<1$ then the second term is positive and dominates the other two for large $M$. If $|x| \geq 1$ then the second term is nonnegative, but the first term is positive and dominates the last one for large $M$.

Finally, we extend $q$ as a 0 -homogeneous symbol in $T^{*} \mathbb{R}^{n}$. Then (17) implies (14). However, insofar (13) holds only for large $x$. To remedy this we observe that (14) is stable with respect to small $C^{1} 0$-homogeneous perturbations of $q$. Thus we can approximate the $q$ we have constructed with a smooth replacement which still satisfies (14).

\section{A CONSTANT COEFFiCIENT Dispersive/LOCAL SMOOTHING ESTIMATE}

The dispersive estimate (10) for $w$ follows from the following more general result:

Theorem 3. Consider the initial value problem

$$
\left\{\begin{array}{l}
P(D) w=f(x, t) \\
w(x, 0)=w_{0}(x)
\end{array}\right.
$$

with $f$ supported in $[0,1] \times B(0,1)$. Then for any admissible couple $(q, r)$ we have

$$
\|w\|_{L^{q}\left(L^{r}\right)} \lesssim\left\|w_{0}\right\|_{L^{2}}+\|f\|_{L^{2}\left([0,1], H^{-1 / 2}\right)} .
$$

This result shows that the Strichartz estimates are compatible with the local smoothing norms introduced by Kenig-Ponce-Vega [12] in connection to the local well-posedness problem for semilinear Schröedinger type equations.

The compact support assumption in time is not needed in dimension $n \geq 2$. However, for $n=1$ the fundamental solution decays only like $t^{-\frac{1}{2}}$, therefore there is an obstruction to (19) coming from the low frequencies in $f$.

With the exception of the end-point result (which corresponds to $q=2$ ) one can give a very simple proof of the Theorem using a lemma of Christ and Kiselev. As observed by Tao, this lemma allows one to reduce inhomogeneous estimates to homogeneous ones; in our case, it reduces the Theorem to the much simpler Lemma 3 below (modulo the homogeneous Strichartz estimates, which are known). For completeness we give the lemma in the formulation which was used in a similar context by Smith-Sogge [16]. 
Lemma 2. Let $X$ and $Y$ be Banach spaces and assume that $K(t, s)$ is a continuous function taking its values in $B(X, Y)$, the space of bounded linear mappings from $X$ to $Y$. Suppose that $\infty \leq a<b \leq \infty$, and set

$$
T f(t)=\int_{a}^{b} K(t, s) f(s) d s, \quad W f(t)=\int_{a}^{t} K(t, s) f(s) d s
$$

Assume that $1 \leq p<q \leq \infty$ and

$$
\|T f\|_{L^{q}([a, b], Y)} \leq C\|f\|_{L^{p}([a, b], X)} .
$$

Then

$$
\|W f\|_{L^{q}([a, b], Y)} \leq C\|f\|_{L^{p}([a, b], X)} .
$$

Then one uses the lemma by setting $X=H^{-1 / 2}\left(\mathbb{R}^{n}\right), Y=L^{r}\left(\mathbb{R}^{n}\right), p=2, q>2$, with $(q, r)$ an admissible couple,

$$
T f(x, t)=\int_{0}^{1} e^{i\left(t-t^{\prime}\right) \Delta} f\left(x, t^{\prime}\right) d t^{\prime}
$$

and

$$
W f(x, t)=\int_{0}^{t} e^{i\left(t-t^{\prime}\right) \Delta} f\left(x, t^{\prime}\right) d t^{\prime} .
$$

In our case we also want to get the endpoint estimates. The idea of the proof is the same, namely to reduce the problem to the corresponding estimates for the homogeneous Schröedinger equation. The argument is somewhat more complicated but it is self-contained.

Proof of Theorem 3. We start with a Lemma in which we compute the regularity of the Fourier transform of $f$ on the characteristic paraboloid $\tau=\xi^{2}$.

Lemma 3. If $f \in L^{2}\left(\mathbb{R}, H^{-1 / 2}\left(\mathbb{R}^{n}\right)\right)$, with support in $[0,1] \times B_{1}$, then

$$
\left\|\hat{f}\left(\xi, \tau=|\xi|^{2}\right)\right\|_{L_{\xi}^{2}} \lesssim\|f\|_{L^{2}\left([0,1], H^{-1 / 2}\right)} .
$$

Taking the Fourier transform of $f$, the estimate would follow from

$$
\left\|\hat{f}\left(\xi, \tau=|\xi|^{2}\right)\right\|_{L_{\xi}^{2}} \lesssim\left\|(1+|\xi|)^{-\frac{1}{2}} \hat{f}\right\|_{L^{2}}+\left\|(1+|\xi|)^{-\frac{1}{2}} \partial_{\xi} \hat{f}\right\|_{L^{2}}+\left\|(1+|\xi|)^{-\frac{1}{2}} \partial_{\tau} \hat{f}\right\|_{L^{2}}
$$

which is equivalent to

$\left\|(1+|\xi|)^{-\frac{1}{2}} \hat{f}\right\|_{L^{2}(K)} \lesssim\left\|(1+|\xi|)^{-\frac{1}{2}} \hat{f}\right\|_{L^{2}}+\left\|(1+|\xi|)^{-\frac{1}{2}} \partial_{\xi} \hat{f}\right\|_{L^{2}}+\left\|(1+|\xi|)^{-\frac{1}{2}} \partial_{\tau} \hat{f}\right\|_{L^{2}}$

where the first norm is taken with respect to the surface measure on the characteristic set $K=\left\{\tau=|\xi|^{2}\right\}$. But this follows easily from the trace theorem. Note that one has to use the $\xi$ derivative for large $\xi$ and the $\tau$ derivative for small $\xi$.

The next step in the proof of the Theorem is to obtain a decomposition of $u$ into a sum of truncated $L^{2}$ solutions to the homogeneous equation modulo a nice remainder. Let $\chi$ be a function of one variable whose Fourier transform is compactly supported and so that $\hat{\chi}-\frac{1}{x-i 0}$ is bounded.Then $\chi$ is smooth and behaves like the Heaviside function at $\pm \infty$. 
Lemma 4. Let $w$ solve the equation

$$
\left(i \partial_{t}-\Delta\right) w=f
$$

where $f$ is supported in $[0,1] \times B(0,1)$. Then there is a decomposition

$$
w=\chi(t) w_{0}+\sum_{j=1}^{n} \chi\left(\mp x_{j}\right) w_{j}^{ \pm}+\tilde{w}
$$

so that the functions $w_{j}^{ \pm}$solve the homogeneous equation $\left(i \partial_{t}-\Delta\right) w_{j}^{ \pm}=0$ and (21)

$$
\left\|w_{0}(0)\right\|_{L^{2}}+\sum_{j=1}^{n}\left\|w_{j}^{ \pm}(0)\right\|_{L^{2}}+\left\|\left((1+|\xi|)^{\frac{1}{2}}+\frac{\left.|\tau-| \xi\right|^{2} \mid}{(1+|\xi|)^{\frac{1}{2}}}\right) \widehat{\widetilde{w}}\right\|_{L^{2}} \lesssim\|f\|_{L^{2}\left(H^{-\frac{1}{2}}\right)} .
$$

To construct the functions $w_{j}$ we start with a nice partition of unit with respect to $\xi$, namely $1=\phi_{0}(\xi)+\sum_{j=1}^{n} \phi_{j}^{ \pm}(\xi)$, so that

$$
\text { supp } \phi_{0} \subset B(0,20), \quad \operatorname{supp} \phi_{j}^{ \pm} \subset\left\{|\xi| \leq \pm 2 \xi_{j}\right\} \backslash B(0,10) .
$$

We set the spatial Fourier transforms of the initial data for $w_{j}$ as

$$
\widehat{w_{0}(0)}(\xi)=\phi_{0}(\xi) \hat{f}\left(\xi, \xi^{2}\right), \quad \widehat{w_{j}^{ \pm}(0)}(\xi)=\phi_{j}^{ \pm}(\xi) \hat{f}\left(\xi, \xi^{2}\right)
$$

Then, by Lemma $3,(21)$ holds for the $w_{j}$ 's. It remains to verify the estimate for $\tilde{w}$. We have

$$
{\widehat{w_{0}}}^{ \pm}(\tau, \xi)=\phi_{0}(\xi) \hat{f}\left(\xi, \xi^{2}\right) \delta_{\tau=\xi^{2}}, \quad{\widehat{w_{j}}}^{ \pm}(\tau, \xi)=\phi_{j}^{ \pm}(\xi) \hat{f}\left(\xi, \xi^{2}\right) \delta_{\tau=\xi^{2}} .
$$

Hence

$$
\widehat{\chi(t) w_{0}}(\tau, \xi)=\phi_{0}(\xi) \hat{f}\left(\xi, \xi^{2}\right) \hat{\chi}\left(\tau-\xi^{2}\right) .
$$

For $j=1, \ldots, n$ we denote by $\xi_{j}^{\prime}$ the vector of all coordinates except $\xi_{j}$. On the paraboloid we write

$$
\xi_{j}=\xi_{j}^{ \pm}\left(\tau, \xi^{\prime}\right)= \pm \sqrt{\tau-\left(\xi_{j}^{\prime}\right)^{2}}
$$

Then

and

$$
\delta_{\tau=\xi^{2}}=\frac{1}{2 \xi_{j}^{ \pm}\left(\xi_{j}^{\prime}, \tau\right)} \delta_{\xi_{j}=\xi_{j}^{ \pm}\left(\xi_{j}^{\prime}, \tau\right)}
$$

$$
\chi \widehat{\left(\mp x_{j}\right)} w_{j}(\tau, \xi)=\frac{\phi_{j}\left(\xi_{j}^{\prime}, \xi_{j}^{ \pm}\left(\tau, \xi_{j}^{\prime}\right)\right)}{2 \xi_{j}^{ \pm}\left(\xi_{j}^{\prime}, \tau\right)} \hat{f}\left(\xi_{j}^{\prime}, \xi_{j}^{ \pm}\left(\tau, \xi_{j}^{\prime}\right), \tau\right) \hat{\chi}\left(\mp\left(\xi_{j}-\xi_{j}^{ \pm}\left(\tau, \xi_{j}^{\prime}\right)\right)\right) .
$$

Now we are in a position to estimate the Fourier transform of $\tilde{w}$. Outside a neighborhood of size 1 of the paraboloid we have $\left|\tau-\xi^{2}\right| \gtrsim(1+|\xi|)$ and

$$
\widehat{\widetilde{w}}=\hat{w}=\hat{f}\left(\tau-\xi^{2}\right)^{-1}
$$

so the conclusion follows. Near the paraboloid, on the other hand, we have

$$
\widehat{\widetilde{w}}=g_{0}+\sum_{j=1}^{n} g_{j}^{ \pm}
$$

where

$$
g_{0}=\phi_{0}(\xi)\left(\frac{\hat{f}(\tau, \xi)}{\left(\tau-\xi^{2}-i 0\right)}-\hat{\chi}\left(\tau-\xi^{2}\right) f\left(\xi, \xi^{2}\right)\right)
$$


$g_{j}^{ \pm}=\phi_{j}^{ \pm}(\xi) \frac{\hat{f}(\tau, \xi)}{\left(\tau-\xi^{2}-i 0\right)}-\frac{\phi_{j}^{ \pm}\left(\xi_{j}^{\prime}, \xi_{j}^{ \pm}\left(\tau, \xi_{j}^{\prime}\right)\right)}{2 \xi_{j}^{ \pm}\left(\xi_{j}^{\prime}, \tau\right)} \hat{f}\left(\xi_{j}^{\prime}, \xi_{j}^{ \pm}\left(\tau, \xi_{j}^{\prime}\right), \tau\right) \hat{\chi}\left(\mp\left(\xi_{j}-\xi_{j}\left(\tau, \xi_{j}^{\prime}\right)\right)\right)$.

We estimate each term separately. For $g_{0}$ we have

$$
g_{0}=\phi_{0}(\xi)\left(\frac{\hat{f}(\xi, \tau)-\hat{f}\left(\xi, \xi^{2}\right)}{\left(\tau-\xi^{2}-i 0\right)}-\left(\hat{\chi}\left(\tau-\xi^{2}\right)-\frac{1}{\left(\tau-\xi^{2}-i 0\right)}\right) f\left(\xi, \xi^{2}\right)\right)
$$

which is bounded in the range $|\xi|, \tau \lesssim 1$ due to our compact support assumption on $f$. It remains to estimate $g_{j}$ in the range

$$
A_{j}^{ \pm}=\left\{|\xi| \leq 2 \pm \xi_{j},|\xi|>10,\left|\xi_{j}-\xi_{j}\left(\tau, \xi_{j}^{\prime}\right)\right| \leq 1\right\} .
$$

We decompose it as before,

$$
\begin{aligned}
g_{j}^{ \pm} & =\phi_{j}^{ \pm}(\xi) \frac{\hat{f}(\tau, \xi)-\hat{f}\left(\tau, \xi_{j}^{\prime}, \xi_{j}^{ \pm}\left(\tau, \xi_{j}^{\prime}\right)\right)}{\left(\tau-\xi^{2}-i 0\right)} \\
& -\left(\frac{\phi_{j}^{ \pm}\left(\xi_{j}^{\prime}, \xi_{j}\left(\tau, \xi_{j}^{\prime}\right)\right)}{2 \xi_{j}\left(\xi_{j}^{\prime}, \tau\right)} \hat{\chi}\left(\mp\left(\xi_{j}-\xi_{j}\left(\tau, \xi_{j}^{\prime}\right)\right)\right)-\frac{\phi_{j}^{ \pm}(\xi)}{\left(\tau-\xi^{2}-i 0\right)}\right) \hat{f}\left(\xi_{j}^{\prime}, \xi_{j}^{ \pm}\left(\tau, \xi_{j}^{\prime}\right), \tau\right) .
\end{aligned}
$$

For the first term we observe that in $A_{j}^{ \pm}$

$$
\left.\left|\tau-\xi^{2}\right| \approx|\xi| \mid \xi_{j}-\xi_{j}^{ \pm}\left(\tau, \xi_{j}^{\prime}\right)\right) \mid .
$$

Then we bound the increment of $\hat{f}$ by its derivative to get

$$
\left\|\phi_{j}^{ \pm}(\xi) \frac{\hat{f}(\tau, \xi)-\hat{f}\left(\tau, \xi_{j}^{\prime}, \xi_{j}^{ \pm}\left(\tau, \xi_{j}^{\prime}\right)\right)}{\left(\tau-\xi^{2}-i 0\right)}\right\|_{L^{2}\left(A_{j}^{ \pm}\right)} \lesssim\left\||\xi|^{-1} \partial_{\xi_{j}} \hat{f}\right\|_{L^{2}\left(A_{j}^{ \pm}\right)} .
$$

Since $f$ has compact support, it follows that $\partial_{\xi_{j}} \hat{f}$ has the same regularity as $\hat{f}$. Then we get the desired estimate.

The bound for the second term in the region $A_{j}$ follows from the $L^{2}\left(H^{-\frac{1}{2}}\right)$ regularity of $f$; indeed, due to the choice of $\hat{\chi}$ it is easy to verify that

$$
\left|\frac{\phi_{j}^{ \pm}\left(\xi_{j}^{\prime}, \xi_{j}^{ \pm}\left(\tau, \xi_{j}^{\prime}\right)\right)}{2 \xi_{j}^{ \pm}\left(\xi_{j}^{\prime}, \tau\right)} \hat{\chi}\left(\mp\left(\xi_{j}-\xi_{j}\left(\tau, \xi_{j}^{\prime}\right)\right)\right)-\frac{\phi_{j}^{ \pm}(\xi)}{\left(\tau-\xi^{2}-i 0\right)}\right| \lesssim \frac{1}{1+|\xi|} .
$$

This concludes the proof of the Lemma.

Now we conclude the proof of the Theorem. We know that the $L^{q}\left(L^{r}\right)$ estimates hold for $L^{2}$ solutions to the homogeneous equation. This gives the correct bound for $\chi(t) w_{0}$ and $\chi\left(\mp x_{j}\right) w_{j}$. It remains to look at $\tilde{w}$. We can use a Littlewood-Paley decomposition to reduce the estimate for $\tilde{w}$ to the case when $\tilde{w}$ is frequency localized in the region $|\xi| \sim \lambda$. Thus we substitute $\widehat{\tilde{w}}$ with $\widehat{\widehat{w}_{\lambda}}=\chi(|\xi| / \lambda) \widehat{\tilde{\tilde{w}}}$, where $\chi$ is a smooth characteristic function of the interval $[1,2]$. Then we foliate its Fourier transform with respect to translated paraboloids,

$$
\widehat{\tilde{w}_{\lambda}}(\xi, \tau)=\int_{\mathbb{R}} \widehat{\tilde{w}_{\lambda}}\left(\xi, \xi^{2}+s\right) \delta_{\tau-\xi^{2}=s} d s .
$$

If we denote by $\tilde{w}_{s, \lambda}$ the solution to the homogeneous equation with initial data $\widehat{\tilde{w}_{s, \lambda}(0)}=\widehat{\widetilde{w}_{\lambda}}\left(\xi, \xi^{2}+s\right)$ then

$$
\tilde{w}_{\lambda}(x, t)=\int_{\mathbb{R}} e^{i s t} \tilde{w}_{s, \lambda}(x, t) d s .
$$


Hence, using the Strichartz estimates for the homogeneous equation,

$$
\left\|\tilde{w}_{\lambda}\right\|_{L^{q}\left(L^{r}\right)} \lesssim \int\left\|\widehat{\tilde{w}}_{\lambda}\left(\xi, \xi^{2}+s\right)\right\|_{L_{\xi}^{2}} d s
$$

From Cauchy-Schwartz with respect to $s$ we get

$$
\|\tilde{w}\|_{L^{q}\left(L^{r}\right)}^{2} \lesssim\left(\int\left(\lambda^{1 / 2}+\lambda^{-1 / 2}|s|\right)^{2}\left|\widehat{\tilde{w}}_{\lambda}\right|^{2}\left(\xi, \xi^{2}+s\right) d \xi d s\right)\left(\int_{s \gtrsim \lambda} \lambda(\lambda+s)^{-2} d s\right)
$$

which after the change of coordinates $\tau=s+|\xi|^{2}$ gives

$$
\left\|\tilde{w}_{\lambda}\right\|_{L^{q}\left(L^{r}\right)}^{2} \lesssim\left\|\widehat{\tilde{w}_{\lambda}}(\xi, \tau)\left(\lambda^{1 / 2}+\left.\lambda^{-1 / 2}|\tau-| \xi\right|^{2} \mid\right)\right\|_{L^{2}}^{2}
$$

Given the bound for $\tilde{w}$ in (21), this concludes our proof.

\section{The localized VARiable COEFFiCient EStimate}

Here we prove that the Strichartz estimates hold locally for the variable coefficient Schröedinger equation, in the context provided by local smoothing.

Theorem 4. Assume that (A1) and (A2) are satisfied. Let $(q, r)$ be an admissible pair. Then for any $v$ supported in a fixed compact set we have

$$
\|v\|_{L^{q}\left(L^{r}\right)} \leq\|v\|_{L^{2} H^{1 / 2}}+\|P v\|_{L^{2} H^{-1 / 2}} .
$$

whenever the right hand side is finite.

This implies (11) after a truncation in time. Note that the first term on the right can be estimated by the second whenever local smoothing holds. For this one needs the nontrapping condition (A3). Our result is formulated in such a way so that we avoid the need for a nontrapping assumption.

The argument is fairly long, so we provide a brief outline here. $\aleph 4.1$ contains several localization type arguments. More precisely, we use a Paley-Litlewood decomposition to reduce the problem to the corresponding dyadic estimates at fixed frequency $\lambda$. Simultaneously we truncate the coefficients of $P$ at frequency $\sqrt{\lambda}$.

In $\aleph 4.2$ we describe the FBI transform and show how to conjugate pseudodifferential operators with respect to it. Conjugating the operator $P$ with respect to the FBI transform in $\aleph 4.3$ we get two ode's in the phase space. One of these ode's is along the gradient flow of $p$ and provides an elliptic estimate away from the characteristic set of $P$ in $\aleph 4.4$. The other one is along the Hamilton flow of $p$ and corresponds to propagation of singularities. In $\aleph 4.5$ we study the regularity of the gradient flow, which in $\aleph 4.6$ yields a nice representation (parametrix) of the function $v$ in terms of certain oscillatory integrals which are reminiscent of Fourier integral operators with complex phase.

The last step is to prove the estimates for the oscillatory integrals, using stationary phase and Stein's complex interpolation theorem. In $\aleph 4.7$ we set up the interpolation argument. The easy part in the interpolation, namely the reduction to a pointwise bound for an oscillatory integral, is carried out in $\aleph 4.8$ and $\aleph 4.9$.

The estimate for the oscillatory integral requires a careful analysis of the regularity of the Hamilton flow, which is done in $\aleph 4.10$. This opens the way for the short range estimate in $\aleph 4.11$ and the long range estimate in $\aleph 4.12$. 
4.1. Reduction to dyadic estimates. Given a smooth function $s$ supported in $\left[\frac{1}{2}, 2\right]$ so that

$$
\sum_{j \in \mathbb{Z}} s\left(2^{-j} x\right)=1
$$

we define the multipliers

$$
s_{\lambda}=s\left(\lambda^{-1}\left(\tau^{2}+\xi^{4}\right)^{\frac{1}{4}}\right)
$$

which are supported in the region at frequency $\lambda$. We also define the multipliers

$$
U_{\lambda}=\sum_{\mu<\lambda} s_{\lambda}
$$

which select the frequencies $\lambda$ and lower. Correspondingly, let

$$
P_{\lambda}=i \partial_{t}-\partial_{i} a_{\lambda}^{i j} \partial_{j}, \quad a_{\lambda}^{i j}=U_{\sqrt{\lambda}} a^{i j}
$$

be the "mollified" operator obtained from $P$ by truncating the coefficients at frequency $\lambda^{\frac{1}{2}}$. The new coefficients $a_{\lambda}^{i j}$ are a small perturbation of $a^{i j}$, in the sense that

$$
\left\|a^{i j}-a_{\lambda}^{i j}\right\|_{L^{\infty}} \lesssim \lambda^{-1}\left(\left\|\left|\partial_{x}^{2} a^{i j}\right|+\left|\partial_{t} a^{i j}\right|\right\|_{L^{\infty}}\right) .
$$

Then we claim that (22) reduces to the corresponding dyadic estimates,

$$
\left\|S_{\lambda} u\right\|_{L^{q}\left(L^{r}\right)} \leq \lambda^{\frac{1}{2}}\left\|S_{\lambda} u\right\|_{L^{2}}+\lambda^{-\frac{1}{2}}\left\|P_{\lambda} S_{\lambda} u\right\|_{L^{2}} .
$$

In order to obtain (22) from (24) we square (24) and sum over $\lambda=2^{j}, j \geq 0$. We get (22) provided we can handle the errors and show that

$$
\sum_{\lambda=2^{j}}\left\|\left(P_{\lambda} S_{\lambda}-S_{\lambda} P\right) u\right\|_{H^{-\frac{1}{2}}}^{2} \lesssim\|u\|_{H^{\frac{1}{2}}}^{2} .
$$

This would in turn follow by summation from

$$
\left\|\left(P_{\lambda} S_{\lambda}-S_{\lambda} P\right) u\right\|_{L^{2}} \lesssim \min \left\{\|u\|_{H^{1}}, \lambda\|u\|_{L^{2}}\right\},
$$

where the two bounds on the right are used for the low $(\lesssim \lambda)$ respectively for the high frequencies in $u$. The first part of (25) reduces to

$$
\left\|a_{\lambda}^{i j} S_{\lambda}-S_{\lambda} a^{i j}\right\|_{L^{2} \rightarrow L^{2}} \lesssim \lambda^{-1}
$$

and further, by (23), to the commutator estimate

$$
\left\|\left[a^{i j}, S_{\lambda}\right]\right\|_{L^{2} \rightarrow L^{2}} \lesssim \lambda^{-1} .
$$

This is a consequence of the following bound for the kernel $K$ of the commutator:

$$
\begin{aligned}
|K(t, x, s, y)| & =\left|\left(a^{i j}(t, x)-a^{i j}(s, y)\right) \lambda^{n+2} \hat{s}\left(\lambda^{2}(t-s), \lambda(x-y)\right)\right| \\
& \lesssim \lambda^{n+1}\left(1+\lambda^{2}|t-s|+\lambda|x-y|^{2}\right)^{-N} .
\end{aligned}
$$

For the second part of (25) we move all the derivatives to the left of the coefficients, so that they are only used at frequency $\lambda$,

$$
\partial_{i} a^{i j} \partial_{j}=\partial_{i} \partial_{j} a^{i j}-\partial_{i}\left(\partial_{j} a^{i j}\right)
$$

and similarly for $a_{\lambda}^{i j}$. Then we need to show that

$$
\left\|a_{\lambda}^{i j} S_{\lambda}-S_{\lambda} a^{i j}\right\|_{L^{2} \rightarrow L^{2}} \lesssim \lambda^{-1}, \quad\left\|\left(\partial_{x} a_{\lambda}^{i j}\right) S_{\lambda}-S_{\lambda}\left(\partial_{x} a^{i j}\right)\right\|_{L^{2} \rightarrow L^{2}} \lesssim 1 .
$$

But the first bound was proved before and the second is trivial. 
4.2. The FBI transform and error estimates. The FBI transform has been an essential tool in the proof of the Strichartz estimates for the wave equation with nonsmooth coefficients in $[19,20,18]$. We plan to use a similar strategy here. The Schrödinger equation, however, has a different scaling. Thus the first step is to introduce a modified FBI transform adapted to this case. Our results here parallel the corresponding results in [19].

The FBI transform of a temperate distribution $f$ in $\mathbb{R} \times \mathbb{R}^{n}$ is a holomorphic function in $\mathbb{C}^{n+1}$ defined as

$$
\left(T_{\lambda} f\right)\left(z_{0}, z\right)=c_{n} \lambda^{\frac{3(n+2)}{4}} \int e^{-\frac{\lambda}{2}(z-y)^{2}} e^{-\frac{\lambda^{2}}{2}\left(z_{0}-s\right)^{2}} f(s, y) d y d s
$$

where $c_{n}=2^{-\frac{n+1}{2}} \pi^{-\frac{3(n+1)}{4}}$. Set

$$
z_{0}=t-i \tau, \quad z=x-i \xi, \quad \Phi\left(z_{0}, z\right)=e^{-\lambda \xi^{2}-\lambda^{2} \tau^{2}} .
$$

Then the operator $T_{\lambda}$ is an isometry from $L^{2}\left(\mathbb{R}^{n+1}\right)$ onto the closed subspace of holomorphic functions in $L_{\Phi}^{2}\left(\mathbb{C}^{n+1}\right)$. One inversion formula is provided by the adjoint operator:

$$
f(s, y)=c_{n} \lambda^{\frac{3(n+2)}{4}} \int \Phi\left(z_{0}, z\right) e^{-\frac{\lambda}{2}(\bar{z}-y)^{2}} e^{-\frac{\lambda^{2}}{2}\left(\bar{z}_{0}-s\right)^{2}}\left(T_{\lambda} f\right)\left(z_{0}, z\right) d z d \bar{z} d z_{0} d \bar{z}_{0} .
$$

The FBI transform of a function $f$ can also be expressed in terms of the Fourier transform of $f$,

$$
T_{\lambda} f\left(z_{0}, z\right)=c_{n} \lambda^{\frac{5(n+2)}{4}} e^{\frac{\lambda}{2} \xi^{2}} e^{\frac{\lambda^{2}}{2} \tau^{2}} \int e^{-\frac{\lambda}{2}(\eta-\xi)^{2}} e^{i \lambda x \eta} e^{-\frac{\lambda^{2}}{2}(\theta-\tau)^{2}} e^{i \lambda^{2} t \tau} \hat{f}\left(\lambda^{2} \theta, \lambda \eta\right) d \eta d \theta .
$$

Given a compactly supported symbol $q(t, x, \tau, \xi)$ we define the rescaled operators

$$
Q_{\lambda}=Q\left(t, x, \frac{D_{t}}{\lambda^{2}}, \frac{D_{x}}{\lambda}\right) .
$$

Then our main result deals with the problem of conjugating the operator $Q_{\lambda}$ with respect to $T_{\lambda}$. We define our candidate for the conjugated operator as

$$
\tilde{Q}_{\lambda}=q(t, x, \tau, \xi)+\frac{1}{-i \lambda} q_{x}\left(\partial_{\xi}-\lambda \xi\right)+\frac{1}{\lambda} q_{\xi}\left(\frac{1}{i} \partial_{x}-\lambda \xi\right) .
$$

Define also the remainder

$$
R_{\lambda, q}=T_{\lambda} Q_{\lambda}-\tilde{Q}_{\lambda} T_{\lambda}
$$

Then our main result is

Theorem 5. Assume that $\partial_{x}^{2} q, \partial_{t} q \in L_{t, x}^{\infty}\left(C_{0}^{\infty}\right)$. Then

$$
\left\|R_{\lambda, q}\right\|_{L^{2} \rightarrow L_{\phi}^{2}} \leq c \lambda^{-1}
$$

and

$$
\left\|\left(\partial_{\xi}-\lambda \xi\right) R_{\lambda, q}\right\|_{L^{2} \rightarrow L_{\phi}^{2}} \leq c \lambda^{-\frac{1}{2}}
$$

This theorem shows that the approximation we consider is precise up to one derivative. The proof is identical to the proof of Theorem 1 in [19]. 


\subsection{Conjugation and the ode's in the phase space. Set}

$$
v_{\lambda}=T_{\lambda} S_{\lambda} u \text {. }
$$

Then we try to get good $L^{2}$ estimates for $v_{\lambda}$. The function $S_{\lambda} u$ can then be recovered from

$$
S_{\lambda} u=T_{\lambda}^{*} \Phi v_{\lambda} .
$$

Observe first that $v_{\lambda}$ is concentrated in the region

$$
U=\left\{|x| \leq 2, \quad \frac{1}{4} \leq|\xi|^{2}+|\tau| \leq 4\right\} .
$$

Indeed, outside this region we have

$$
\left\|v_{\lambda}\right\|_{L_{\text {के }}^{2}\left(U^{c}\right)} \leq e^{-c \lambda}\left\|S_{\lambda} u\right\|_{L^{2}}
$$

which is a straightforward consequence of the representation formula (27). Hence it suffices to get good estimates for $v_{\lambda}$ in the region $U$.

Start with $S_{\lambda} u$ as in (24), i.e. satisfying

$$
\lambda^{\frac{1}{2}} S_{\lambda} u \in L^{2}, \quad \lambda^{-\frac{1}{2}} P_{\lambda} S_{\lambda} u \in L^{2} .
$$

Since the coefficients of $P$ are $C^{2}$, we can use Theorem 5 for $v_{\lambda}$ in $U$. Note that $P$ is a second order operator, therefore compared with Theorem 5 we need another $\lambda^{2}$ factor in the conjugation. We get

$$
\lambda^{\frac{1}{2}} v_{\lambda} \in L_{\Phi}^{2}, \quad \lambda^{\frac{3}{2}} \tilde{P}_{\lambda} v_{\lambda} \in L_{\Phi}^{2}
$$

and also

$$
\left(\partial_{\xi}-\lambda \xi\right) v_{\lambda} \in L_{\Phi}^{2}, \quad \lambda\left(\partial_{\xi}-\lambda \xi\right) \tilde{P}_{\lambda} v_{\lambda} \in L_{\Phi}^{2}
$$

Here

$$
\tilde{P}_{\lambda}=p(t, x, \tau, \xi)+\frac{1}{-i \lambda} p_{x}\left(\partial_{\xi}-\lambda \xi\right)+\frac{1}{\lambda} p_{\xi}\left(\frac{1}{i} \partial_{x}-\lambda \xi\right) .
$$

Since the operators $i\left(\partial_{\xi}-\lambda \xi\right)$ and $(\partial-i \lambda \xi)$ coincide on holomorphic functions, combining the second relation in (28) with the first in (29) we get

$$
\lambda p v_{\lambda} \in L_{\Phi}^{2} .
$$

Away from the paraboloid $K=\{p=0\}$ this yields an additional $\lambda^{\frac{1}{2}}$ gain for the $L^{2}$ norm of $v_{\lambda}$ compared to (28). This translates into a $1 / 2$ derivative gain in the physical space, which suffices for our estimates by the Sobolev embeddings. Hence it suffices to study $v_{\lambda}$ further in a small neighborhood of the characteristic set $K=\{p(t, x, \tau, \xi)=0\}$ in $U$.

To get good $L^{2}$ estimates for $v_{\lambda}$ it is useful to eliminate first the weight $\Phi$ using the substitution

$$
w=\Phi^{\frac{1}{2}} v_{\lambda} .
$$

Also set

$$
g=\lambda \Phi^{\frac{1}{2}} \tilde{P}_{\lambda} v_{\lambda}
$$

Then from (28), (29) we get

$$
\lambda^{\frac{1}{2}} w \in L^{2}, \quad \lambda^{\frac{1}{2}} g \in L^{2}
$$

respectively

$$
\partial_{\xi} w \in L^{2}, \quad \partial_{\xi} g \in L^{2} .
$$


Using the trace theorem, this yields

$$
\lambda^{\frac{1}{4}} w \in L^{2}(K), \quad \lambda^{\frac{1}{4}} g \in L^{2}(K) .
$$

The relation between $w$ and $g$ is obtained by conjugating $\tilde{P}_{\lambda}$ with respect to $\Phi$, i.e. by replacing $\partial_{\xi}-\lambda \xi$ by $\partial_{\xi}$ :

$$
\left[\lambda p+\frac{1}{-i} p_{x} \partial_{\xi}+p_{\xi}\left(\frac{1}{i} \partial_{x}-\lambda \xi\right)\right] w=g .
$$

On the other hand, since $v_{\lambda}$ is holomorphic, the operators $\frac{1}{i} \partial_{x}-\lambda \xi$ and $\partial_{\xi}-\lambda \xi$ acting on it are interchangeable. After the conjugation with respect to $\Phi^{\frac{1}{2}}$, this implies that the operators $\frac{1}{i} \partial_{x}-\lambda \xi$ and $\partial_{\xi}$ are interchangeable when acting on $w$. Consequently, from (34) we get a second equation, namely

$$
\left[\lambda p+\frac{1}{-i} p_{x}\left(\frac{1}{i} \partial_{x}-\lambda \xi\right)+p_{\xi} \partial_{\xi}\right] w=g .
$$

The first equation is an ode along the Hamilton flow of $p$, while the second equation is an ode along the gradient curves of $p$. Our strategy is now to use the (34) to obtain good estimates for $w$ on the characteristic paraboloid $K$, and then to use (35) to obtain good decay rates away from the paraboloid.

4.4. Estimates away from the paraboloid. We use (34) and (35) to decompose $w$ into two parts,

$$
w=w_{1}+w_{2}
$$

where $w_{1}$ solves the inhomogeneous equation

$$
\left[\lambda p+p_{x}\left(\partial_{x}-i \lambda \xi\right)+p_{\xi} \partial_{\xi}\right] w_{1}=g, \quad w_{1 \mid K}=0
$$

and $w_{2}$ solves the homogeneous equation

$$
\left[\lambda p+p_{x}\left(\partial_{x}-i \lambda \xi\right)+p_{\xi} \partial_{\xi}\right] w_{2}=0, \quad w_{2 \mid K}=w .
$$

Correspondingly we split $S_{\lambda} u$ into $u_{1}+u_{2}$ with

$$
u_{i}=T_{\lambda}^{*} \Phi^{\frac{1}{2}} w_{i} .
$$

We claim that $w_{1}$ satisfies the following bound

$$
\lambda w_{1} \in L^{2} .
$$

This implies that $u_{1}$ is one half derivative more regular than $S_{\lambda} u$, which is exactly what is needed in order to get the $L^{q}\left(L^{r}\right)$ bounds in the Strichartz estimates simply from Sobolev embeddings.

Since $\lambda^{\frac{1}{2}} g \in L^{2},(38)$ would follow from an estimate of the form

$$
\left\|w_{1}\right\|_{L^{2}} \lesssim \lambda^{-\frac{1}{2}}\|g\|_{L^{2}}
$$

It suffices to do this in the region $p>0$. The argument in the region $p<0$ is symmetric. Let $\phi$ be a positive nonincreasing function to be chosen later. We multiply the equation (36) by $\lambda^{-\frac{1}{2}} \phi\left(\lambda^{\frac{1}{2}} p\right) \bar{w}_{1}$ and integrate by parts. This gives

$$
2 \lambda^{-\frac{1}{2}} \Re \int \phi\left(\lambda^{\frac{1}{2}} p\right) \bar{w}_{1} g d x d \xi=\int \psi(x, \xi)\left|w_{1}\right|^{2} d x d \xi,
$$

where

$$
\psi(x, \xi)=2 \lambda^{\frac{1}{2}} p \phi\left(\lambda^{\frac{1}{2}} p\right)-\phi^{\prime}\left(\lambda^{\frac{1}{2}} p\right)|\nabla p|^{2}-\phi\left(\lambda^{\frac{1}{2}} p\right) \lambda^{-\frac{1}{2}} \Delta_{x, \xi} p .
$$


This implies (39) provided we can choose the function $\phi$ so that

$$
c\left(1+\phi^{2}(p)\right)+C \phi(p) \leq p \phi(p)-\phi^{\prime}(p)
$$

for a large constant $C$ and some small constant $c$. But this is easily achieved if we take for instance

$$
\phi(p)=\max \left\{e^{2 C(1-p)}, 1\right\} .
$$

Note that this integration by parts argument requires in effect just one derivative for the coefficients of $P$, together with the fact that they are frequency localized in a ball of radius $\sqrt{\lambda}$. This suffices in order to guarantee that $\Delta_{x} p$ has size at most $\lambda^{\frac{1}{2}}$.

4.5. The gradient flow. The next step in our analysis is to estimate $w_{2}$ using (37). To achieve that we need to compute the regularity of the gradient flow of $p$ with respect to the $(x, \xi)$ variables. Throughout this analysis $(t, \tau)$ are fixed. This is related to the infinite speed of propagation phenomena.

Suppose we start with initial data $(t, x, \tau, \xi)$ on the characteristic set $K=\{p=$ $0\}$. Denote by $q$ the natural parameter along the flow, chosen so that $q=0$ on $K$. Set $\left(x_{q}, \xi_{q}\right)$ the image of $(x, \xi)$ along the flow. Then $\left(x_{q}, \xi_{q}\right)$ solve the equations

$$
\left\{\begin{array}{cc}
\partial_{q} x_{q}=p_{x}\left(x_{q}, \xi_{q}\right) & x(0)=x \\
\partial_{q} \xi_{q}=p_{\xi}\left(x_{q}, \xi_{q}\right) & \xi(0)=\xi
\end{array}\right.
$$

The following result describes the regularity of the gradient flow. Although our operator $P$ has $C^{2}$ coefficients, in effect $C^{1}$ suffices for the arguments involving the gradient flow.

Theorem 6. Assume that $P$ has $C^{1}$ coefficients with Fourier transform supported in $B(0, \sqrt{\lambda})$. Then

$$
\begin{aligned}
\left|\partial_{x}^{\alpha} \partial_{\xi}^{\beta} x_{q}\right| & \leq c_{\alpha, \beta} \lambda^{\frac{|\alpha|-1}{2}} e^{c_{\alpha \beta} \sqrt{\lambda}|q|} & & |\alpha|+|\beta|>0 \\
\left|\partial_{x}^{\alpha} \partial_{\xi}^{\beta}\left(\xi_{q}-\xi\right)\right| & \leq c_{\alpha, \beta} \lambda^{\frac{|\alpha|-1}{2}} e^{c_{\alpha \beta} \sqrt{\lambda}|q|} & & |\alpha|+|\beta|>0 .
\end{aligned}
$$

Thus the gradient flow blows up exponentially on the $\lambda^{\frac{1}{2}}$ scale. This is, however, compensated by the fact that the fundamental solution to (37) exhibits Gaussian decay on precisely the same scale.

Proof. The linearization of the gradient flow is given by the system

$$
\left\{\begin{array}{l}
\partial_{q} y=p_{x x} y+p_{x \xi} \eta \\
\partial_{q} \eta=p_{\xi x} y+p_{\xi \xi} \eta
\end{array}\right.
$$

This implies the inequalities

$$
\left\{\begin{array}{l}
\left|\partial_{q} y\right| \leq c(\sqrt{\lambda}|y|+|\eta|) \\
\left|\partial_{q} \eta\right| \leq c(|y|+|\eta|) .
\end{array}\right.
$$

If we take $(y, \eta)=\left(\partial_{x} x_{q}, \partial_{x} \xi_{q}\right)$ then the Cauchy data in (40) is $(I, 0)$. Thus we obtain the bounds

$$
\left|\partial_{x} x_{q}\right| \leq e^{c \sqrt{\lambda}|q|}, \quad\left|\partial_{x} \xi_{q}\right| \leq \lambda^{-\frac{1}{2}} e^{c \sqrt{\lambda}|q|} .
$$

If we take $(y, \eta)=\left(\partial_{\xi} x_{q}, \partial_{\xi} \xi_{q}\right)$ then the Cauchy data in $(40)$ is $(0, I)$. Then we get the bounds

$$
\left|\partial_{\xi} x_{q}\right| \leq \lambda^{-\frac{1}{2}} e^{c \sqrt{\lambda}|q|}, \quad\left|\partial_{\xi}\left(\xi_{q}-\xi\right)\right| \leq \lambda^{-\frac{1}{2}} e^{c \sqrt{\lambda}|q|} .
$$


The rest follows by induction.

4.6. The oscillatory integral for $u_{2}$. The function $u_{2}$ (which we need to estimate) can be expressed in terms of $w_{2}$, and further in terms of the trace of $w$ on the paraboloid. This computation is carried out in the following theorem:

Theorem 7. Assume that $P$ has $C^{1}$ coefficients frequency localized in $B(0, \sqrt{\lambda})$. Then we have

$$
u_{2}=\lambda^{-\frac{1}{2}} V_{\lambda} \delta_{K} w
$$

where $V_{\lambda}$ is an integral operator,

$\left(V_{\lambda} w\right)(s, y)=\lambda^{\frac{3(n+2)}{4}} \int e^{i \lambda \xi(y-x)} e^{i \lambda^{2} \tau(s-t)} e^{-\frac{\lambda^{2}}{2}(t-s)^{2}} G(t, x, y, \xi) w(t, x, \tau, \xi) d x d \xi d t$ with a kernel $G$ satisfying

$$
\left|\partial_{x}^{\alpha} \partial_{\xi}^{\beta} G(t, x, y, \xi)\right| \leq c_{\alpha, \beta} \lambda^{\frac{|\alpha|}{2}} e^{-c \lambda(x-y)^{2}} .
$$

Proof. We have

$$
u_{2}(s, y)=\lambda^{\frac{3(n+2)}{4}} \int e^{i \lambda \xi(x-y)} e^{i \lambda^{2} \tau(s-t)} e^{-\frac{\lambda^{2}}{2}(t-s)^{2}} e^{-\frac{\lambda}{2}(x-y)^{2}} w_{2}(t, x, \tau, \xi) d t d x d \tau d \xi
$$

Now we choose $q=0$ on the characteristic set $K=\{\tau=a(t, x, \xi)\}$. Then $(t, x, \xi, q)$ can be interpreted locally as a new set of coordinates by

$$
(t, x, \xi, q) \rightarrow\left(t, x_{q}, \xi_{q}, a(t, x, \xi)\right) .
$$

In these new coordinates we write

$$
\begin{aligned}
u_{2}(s, y)= & \lambda^{\frac{3(n+2)}{4}} \int e^{i \lambda \xi_{q}\left(x_{q}-y\right)} e^{i \lambda^{2} a(t, x, \xi)(s-t)} e^{-\frac{\lambda^{2}}{2}(t-s)^{2}} e^{-\frac{\lambda}{2}\left(x_{q}-y\right)^{2}} \\
& w_{2}\left(t, x_{q}, a(t, x, \xi), \xi_{q}\right) d t d x_{q} d a(t, x, \xi) d \xi_{q}
\end{aligned}
$$

Since $w_{2}$ solves the ode (37) we can represent it as

$$
w_{2}\left(t, x_{q}, a(t, x, \xi), \xi_{q}\right)=F(t, x, \xi, q) w(t, x, a(t, x, \xi), \xi)
$$

where $F$ is the solution to the homogeneous equation

$$
\left[\partial_{q}+\lambda\left(p\left(t, x_{q}, a(t, x, \xi), \xi_{q}\right)-i p_{x}\left(t, x_{q}, a(t, x, \xi), \xi_{q}\right) \cdot \xi_{q}\right)\right] F=0 \quad F(t, x, \xi, 0)=1 .
$$

After the change of variable, $u_{2}$ has the form

$$
\begin{aligned}
u_{2}(s, y)= & \lambda^{\frac{3(n+2)}{4}} \int e^{i \lambda \xi_{q}\left(x_{q}-y\right)} e^{i \lambda^{2} a(t, x, \xi)(s-t)} e^{-\frac{\lambda^{2}}{2}(t-s)^{2}} e^{-\frac{\lambda}{2}\left(x_{q}-y\right)^{2}} \\
& F(t, x, \xi, q) \frac{\partial\left(x_{q}, a(t, x, \xi), \xi_{q}\right)}{\partial(x, \xi, q)} w(t, x, a(t, x, \xi), \xi) d t d x d \xi d q
\end{aligned}
$$

or, recombining the exponents,

$$
\begin{aligned}
u_{2}(s, y)= & \lambda^{\frac{3(n+2)}{4}} \int_{K} \int_{\mathbb{R}} e^{i \lambda \xi(x-y)} e^{i \lambda^{2} \tau(s-t)} e^{-\frac{\lambda^{2}}{2}(t-s)^{2}} e^{i \lambda\left(\xi_{q}-\xi\right)\left(x_{q}-y\right)} e^{-\frac{\lambda}{2}\left(x_{q}-y\right)^{2}} \\
& e^{i \lambda \xi\left(x_{q}-x\right)} F(t, x, \xi, q) \frac{\partial\left(x_{q}, \tau, \xi_{q}\right)}{\partial(x, \xi, q)} w_{2}(t, x, \tau, \xi) d t d x d \xi d q .
\end{aligned}
$$

Then

$$
G(t, x, \xi, y)=\lambda^{\frac{1}{2}} \int_{\mathbb{R}} e(t, x, \xi, y, q) d q
$$


where

$$
e(x, \xi, y, q)=e^{i \lambda\left(\xi_{q}-\xi\right)\left(x_{q}-y\right)} e^{-\frac{\lambda}{2}\left(x_{q}-y\right)^{2}} e^{i \lambda \xi\left(x_{q}-x\right)} F(t, x, \xi, q) \frac{\partial\left(x_{q}, a(t, x, \xi), \xi_{q}\right)}{\partial(x, \xi, q)} .
$$

Hence in order to obtain (41) it suffices to show that

$$
\left|\partial_{x}^{\alpha} \partial_{\xi}^{\beta} e(x, \xi, y, q)\right| \leq c_{\alpha, \beta} \lambda^{\frac{|\alpha|}{2}} e^{-c \lambda(x-y)^{2}} e^{-c \lambda q^{2}} .
$$

This, in turn, would follow from

$$
\begin{gathered}
\left|\partial_{x}^{\alpha} \partial_{\xi}^{\beta} e^{i \lambda\left(\xi_{q}-\xi\right)\left(x_{q}-y\right)} e^{-\frac{\lambda}{2}\left(x_{q}-y\right)^{2}}\right| \leq c_{\alpha, \beta} \lambda^{\frac{|\alpha|}{2}} e^{-c \lambda\left(x_{q}-y\right)^{2}} e^{c \sqrt{\lambda} q} \\
\left|\partial_{x}^{\alpha} \partial_{\xi}^{\beta} \frac{\partial\left(x_{q}, a(t, x, \xi), \xi_{q}\right)}{\partial(x, \xi, q)}\right| \leq c_{\alpha, \beta} \lambda^{\frac{|\alpha|}{2}} e^{c \sqrt{\lambda} q} \\
\left|\partial_{x}^{\alpha} \partial_{\xi}^{\beta} e^{i \lambda \xi\left(x_{q}-x\right)} F(t, x, \xi, q)\right| \leq c_{\alpha, \beta} \lambda^{\frac{|\alpha|}{2}} e^{-c \lambda q^{2}} .
\end{gathered}
$$

The bounds (42) and (43) can be easily obtained from Theorem 6 . For (44) observe that

therefore

$$
F(t, x, \xi, q)=e^{\lambda \int_{0}^{q} i p_{x}\left(t, x_{\tilde{q}}, \tau, \xi_{\tilde{q}}\right) \xi_{\tilde{q}}-p\left(t, x_{\tilde{q}}, \tau, \xi_{\tilde{q}}\right) d \tilde{q}}
$$

$$
e^{i \lambda \xi\left(x-x_{q}\right)} F(t, x, \xi, q)=e^{\lambda \int_{0}^{q} i p_{x}\left(t, x_{\tilde{q}}, \tau, \xi_{\tilde{q}}\right)\left(\xi_{\tilde{q}}-\xi\right)-p\left(t, x_{\tilde{q}}, \tau, \xi \tilde{q}\right) d \tilde{q}}
$$

Since $p \approx q$ we obtain the desired Gaussian decay, therefore it remains to show that

$$
\lambda\left|\partial_{x}^{\alpha} \partial_{\xi}^{\beta}\left(i p_{x}\left(t, x_{q}, \tau, \xi_{q}\right)\left(\xi_{q}-\xi\right)-p\left(t, x_{q}, \tau, \xi_{q}\right)\right)\right| \leq c_{\alpha, \beta} \lambda^{\frac{|\alpha|+1}{2}} e^{c \sqrt{\lambda} q} .
$$

This again follows from Theorem 6 .

If we use the equation (34) on $K$ then we get

$$
\left.\left[-p_{x} \partial_{\xi}+p_{\xi} \partial_{x}-i \lambda p_{\xi} \xi\right)\right] w=-i g
$$

Furthermore, on $K$ we have

$$
p_{\xi} \xi=a_{\xi} \xi=2 a=2 \tau
$$

therefore

$$
\left(H_{p}-2 i \lambda \tau\right) w=-i g .
$$

Taking into account the bound (33) for the trace of $g$ on the paraboloid, we need to prove the estimate

$$
\left\|V_{\lambda} \delta_{K} w\right\|_{L^{q}\left(L^{r}\right)} \leq \lambda^{\frac{3}{4}}\left\|\left(H_{p}-2 i \lambda \tau\right) w\right\|_{L^{2}(K)}
$$

for all $w$ supported in $K \cap U$. Note that, since $w$ is compactly supported, the operator $H_{p}-2 i \lambda \tau$ can be replaced by $H_{p}-2 i \lambda \tau+1$.

Given $(t, x, \tau, \xi)$ on $K$ we denote by $\left(t, x_{h}, \tau, \xi_{h}\right)$ its image along the Hamilton flow ( $t$ and $\tau$ rest unchanged). Then (45) is equivalent to

Theorem 8. Let $\alpha(x, \xi)$ be a smooth compactly supported function, which is 0 near $\xi=0$ and 1 in $1 / 4 \leq|\xi| \leq 4$. Then

$$
\left\|V_{\lambda} \alpha(x, \xi) \delta_{K} L\right\|_{L^{2}(K) \rightarrow L^{q}\left(L^{r}\right)} \lesssim \lambda^{\frac{3}{4}}
$$

where $L$ is the operator along the Hamilton flow with kernel

$$
L(h, \tilde{h})=e^{2 i \lambda \tau(h-\tilde{h})} \operatorname{sgn}(h-\tilde{h}) e^{-|h-\tilde{h}|}
$$

which is an inverse for $H_{p}-2 i \lambda \tau+1$. 
This is further equivalent to the corresponding bound for the operator

$$
Z=\left(V_{\lambda} \alpha L\right)\left(V_{\lambda} a L\right)^{*}=V_{\lambda} \alpha L L^{*} \delta_{K} \alpha V_{\lambda}^{*},
$$

namely

$$
\|Z\|_{L^{q^{\prime}\left(L^{r^{\prime}}\right) \rightarrow L^{q}\left(L^{r}\right)}} \lesssim \lambda^{\frac{3}{2}}
$$

The operator $L L^{*}$ is an integral operator along bicharacteristics, with kernel

$$
l(h, \tilde{h})=e^{2 i \lambda \tau(h-\tilde{h})} e^{-|h-\tilde{h}|} .
$$

Next we use complex interpolation to prove the estimate (48) for the non-endpoint case $2<r \leq q$. Then, due to (a simple modification of) Theorem 4 in [19], the endpoint estimate (corresponding to $r=2$ ) follows from the non-endpoint estimate and the bound for the kernel $H_{Z}$ of $Z$,

$$
\left|H_{Z}(s, y, \tilde{s}, \tilde{y})\right| \leq c \lambda^{n+\frac{3}{2}}\left(1+\lambda^{2}|s-\tilde{s}|\right)^{-\frac{n}{2}}
$$

which is a special case of (51) (or a simple consequence of (54)).

4.7. The complex interpolation. Here we set up the complex interpolation argument in order to prove (48) for $2<r \leq q$. First we need to break our operator into a short range and a long range part with respect to time, on the scale of $\lambda^{-3 / 2}$. Let $\chi$ be a smooth function, compactly supported and equal to 1 in a neighborhood of the origin. Then for $\epsilon>0$ we set

$$
\chi_{\epsilon}(x)=\chi(\epsilon x) .
$$

Given an integral operator $W$ with kernel $K(s, \tilde{s}, y, \tilde{y})$ and a smooth, bounded function $\chi$ we denote by $W_{\epsilon}$ the operator with kernel $K(s, \tilde{s}, y, \tilde{y}) \chi_{\epsilon}(s-\tilde{s})$.

Now consider two analytic family of operators

$$
\begin{aligned}
& W_{\theta}^{1}=V_{\lambda} \alpha p^{-\theta}(t, x, \tau, \xi) L_{\theta}^{1} \alpha V_{\lambda}^{*} \\
& W_{\theta}^{2}=V_{\lambda} \alpha p^{-\theta}(t, x, \tau, \xi) L_{\theta}^{2} \alpha V_{\lambda}^{*}
\end{aligned}
$$

where $L_{\theta}^{1}, L_{\theta}^{2}$ are the operators along the Hamilton flow of $P$ with kernels

$$
l_{\theta}^{1}=\theta h^{\theta-1} e^{-|h|} e^{2 i h \lambda a(t, x, \xi)}, \quad l_{\theta}^{2}=\theta h^{\theta-1} e^{-|h|} e^{2 i h \lambda \tau} .
$$

and $p^{\theta}$ is the distribution

$$
p^{-\theta}=(p+i 0)^{-\theta}-(p-i 0)^{-\theta} .
$$

Then

$$
Z=W_{1}^{1}=W_{1}^{2} .
$$

We combine these two families, using $W_{\theta}^{1}$ for the short range and $W_{\theta}^{2}$ for the long range,

$$
Z_{\theta}=W_{\theta, \lambda^{\frac{3}{2}}}^{1}+W_{\theta}^{2}-W_{\theta, \lambda^{\frac{3}{2}}}^{2},
$$

so that we still have $Z_{1}=Z$.

Choose $q_{1}, \theta_{1}$ so that the points

$$
\left(\frac{1}{2}, \frac{1}{2}, 0\right), \quad\left(\frac{1}{q}, \frac{1}{r}, 1\right), \quad\left(\frac{1}{q_{1}}, 0, \theta_{1}\right)
$$

are collinear. Then our estimate follows by interpolation from the following:

$$
\begin{gathered}
\left\|Z_{\theta}\right\|_{L^{2} \rightarrow L^{2}} \leq 1 \quad \Re \theta=0 \\
\left\|Z_{\theta}\right\|_{L^{q_{1}^{\prime}}\left(L^{1}\right) \rightarrow L^{q_{1}}\left(L^{\infty}\right)} \leq \lambda^{\frac{3}{2} \Re \theta} \quad \Re \theta=\theta_{1} .
\end{gathered}
$$


4.8. The $L^{2}$ estimate. We first prove that $W_{\theta}^{1}, W_{\theta}^{2}$ are $L^{2}$ bounded. It is easy to see that for $\Re \theta=0$ the operators $L_{\theta}^{j}$ are $L^{2}$ bounded. Hence it remains to prove that $V_{\lambda}$ is $L^{2}$ bounded.

The kernel of $V_{\lambda} V_{\lambda}^{*}$ is

$$
\begin{aligned}
K_{0}(s, \tilde{s}, y, \tilde{y}) & =\lambda^{\frac{3(n+2)}{2}} \int e^{i \lambda(y-\tilde{y}) \xi} e^{i \lambda^{2} \tau(s-\tilde{s})} e^{-\frac{\lambda^{2}}{2}\left((t-s)^{2}+(t-\tilde{s})^{2}\right)} \\
& \times G(t, x, \xi, y) G(t, x, \xi, \tilde{y}) \alpha^{2}(t, x, \tau, \xi) d t d x d \tau d \xi
\end{aligned}
$$

Integration in $(\tau, \xi)$ yields

$$
\begin{aligned}
\left|K_{0}(s, \tilde{s}, y, \tilde{y})\right| & \leq \lambda^{\frac{3(n+2)}{2}} \int \frac{e^{-c \lambda(y-x)^{2}} e^{-c \lambda(\tilde{y}-x)^{2}} e^{-\frac{\lambda^{2}}{2}\left((t-s)^{2}+(t-\tilde{s})^{2}\right)}}{\left(1+\lambda|y-\tilde{y}|+\lambda^{2}|s-\tilde{s}|\right)^{N}} d x d t \\
& \leq \lambda^{n+2}\left(1+\lambda|y-\tilde{y}|+\lambda^{2}|s-\tilde{s}|\right)^{-N}
\end{aligned}
$$

and the $L^{2}$ boundedness follows.

To transfer the result to $Z_{\theta}$ we use the following simple Lemma, proved in [20]:

Lemma 5. Let $T: L^{2} \rightarrow L^{2}$ be a bounded operator. Then the operators $T_{\epsilon}$ are bounded from $L^{2}$ into $L^{2}$, uniformly in $\epsilon>0$.

4.9. The $L^{q_{1}^{\prime}}\left(L^{1}\right) \rightarrow L^{q_{1}}\left(L^{\infty}\right)$ estimate. This would follow from the following bound on the kernel of $Z_{\theta}$,

$$
\left|K_{\theta, Z}(s, \tilde{s}, y, \tilde{y})\right| \leq c \lambda^{\frac{3}{2} \theta_{1}}|s-\tilde{s}|^{-\frac{2}{q_{1}}}, \quad \Re \theta=\theta_{1} .
$$

Observe that

This follows from the relation

$$
\frac{2}{q_{1}}=\frac{n+2}{2}-\theta_{1}
$$

$$
\frac{2}{q}+\frac{n}{r}=\frac{n+2}{2}-\theta
$$

which, by (6), holds for the first two points in (49) therefore it must also hold for the third.

Then it suffices to prove that

$$
\left|K_{\theta, Z}(s, \tilde{s}, y, \tilde{y})\right| \lesssim c \lambda^{n+2-\frac{\Re \theta}{2}}\left(1+\lambda^{2}|s-\tilde{s}|\right)^{-\left(\frac{n+2}{2}-\Re \theta\right)} \quad 1 \leq \Re \theta \leq \frac{n+1}{2}
$$

which requires that

$$
\left|K_{W_{\theta}^{1}}(s, \tilde{s}, y, \tilde{y})\right| \lesssim c \lambda^{n+2-\frac{\Re \theta}{2}}\left(1+\lambda^{2}|s-\tilde{s}|\right)^{-\left(\frac{n+2}{2}-\Re \theta\right)} \quad|s-\tilde{s}| \leq c \lambda^{-\frac{3}{2}}
$$
respectively

$$
\left|K_{W_{\theta}^{2}}(s, \tilde{s}, y, \tilde{y})\right| \lesssim \lambda^{n+2-\frac{\Re \theta}{2}}\left(1+\lambda^{2}|s-\tilde{s}|\right)^{-\left(\frac{n+2}{2}-\Re \theta\right)} \quad|s-\tilde{s}| \geq c \lambda^{-\frac{3}{2}} .
$$

Our aim is to eliminate the parameter $\theta$ and reduce these estimates to simpler ones. Towards this goal denote by $F^{h}$ the translation by $h$ along the Hamilton flow. Then introduce the kernel $H^{h}$ of the operator

$$
Z^{h}=V_{\lambda} a F^{h} e^{2 i \lambda h a} \delta_{p(t, x, \tau, \xi)=0} a V_{\lambda}^{*} .
$$

Theorem 9. The kernels $H^{h}$ satisfy the following estimate:

(54) $\left|H^{h}(s, \tilde{s}, y, \tilde{y})\right| \lesssim \lambda^{n+2}\left(1+\lambda^{2}|s-\tilde{s}|\right)^{-\frac{n}{2}}\left((1+\sqrt{\lambda}|\lambda(\tilde{s}-s)-h|)^{-N} \cdot+e^{-c h^{2} \lambda}\right)$ 
Now we prove that (54) implies (52) and (53). Observe that as a particular case of (51) for $\theta=1$ we also obtain a bound for the kernel $H_{Z}$ of $Z$ :

Corollary 1. The kernel $H_{Z}$ of the operator $Z$ defined in (47) satisfies the bound

$$
\left|H_{Z}(s, y, \tilde{s}, \tilde{y})\right| \leq c \lambda^{n+2-\frac{1}{2}}\left(1+\lambda^{2}|s-\tilde{s}|\right)^{-\frac{n}{2}} .
$$

To prove that (54) implies (52) we claim that the kernel $K_{W_{\theta}^{1}}$ can be represented as

$$
K_{W_{\theta}^{1}}(s, \tilde{s}, y, \tilde{y})=\left(\lambda^{2}(s-\tilde{s})\right)^{\theta-1} \int \theta h^{\theta-1} e^{-|h|} H^{h}(s, \tilde{s}, y, \tilde{y}) d h .
$$

Indeed, observe first that

$$
L_{\theta}^{1}=\int \theta h^{\theta-1} e^{-|h|} F^{h} e^{2 i h \lambda a} d h .
$$

Then it remains to verify that

$$
V_{\lambda} a p^{-\theta} F^{h} e^{2 i h \lambda a} a V_{\lambda}(s, \tilde{s})=\left(\lambda^{2}(s-\tilde{s})\right)^{\theta-1} V_{\lambda} a \delta_{K} F^{h} e^{2 i h \lambda a} a V_{\lambda}(s, \tilde{s}) .
$$

For this we take advantage of the special form of the symbol,

$$
p(x, \xi)=\tau-a(t, x, \xi) .
$$

and also of the fact that $t, \tau, a(x, \xi, \tau)$ are preserved along the Hamilton flow. Then the only part which depends on $\tau$ in the integral expression for the kernel of $V_{\lambda} a p^{-\theta} e^{i h \lambda a} F^{h} a V_{\lambda}(s, \tilde{s})$ is

$$
\begin{aligned}
I & =\int e^{i \lambda^{2} \tau(s-\tilde{s})}(\theta-1)(\tau-a(t, x, \xi))^{-\theta} d \tau \\
& =\left(\lambda^{2}(s-\tilde{s})\right)^{\theta-1} e^{i \lambda^{2} a(t, x, \xi)(s-\tilde{s})} \\
& =\left(\lambda^{2}(s-\tilde{s})\right)^{\theta-1} \int e^{i \lambda^{2} \tau(s-\tilde{s})} \delta_{K} d \tau .
\end{aligned}
$$

This concludes the proof of (56). Combined with (54) this gives (52) provided that we verify the inequality

$$
\int|h|^{\theta-1}\left((1+\sqrt{\lambda}|\lambda s-h|)^{-N}+e^{-c h^{2} \lambda}\right) d h \leq c \lambda^{-\frac{\theta}{2}}
$$

for

But this is straightforward.

$$
1 \leq \theta \leq \frac{n+1}{2}, \quad|s|<\lambda^{-\frac{3}{2}}
$$

To prove that (54) implies (53) we compute in a similar manner

$$
K_{W_{\theta}^{2}}(s, \tilde{s}, y, \tilde{y})=\left(\lambda^{2}(s-\tilde{s})-\lambda h\right)^{\theta-1} \int h^{\theta-1} e^{-|h|} H^{h}(s, \tilde{s}, y, \tilde{y}) d h
$$

Combined with (54) this gives (53) provided that we verify the inequality

$$
\left.\int|h|^{\theta-1}\left|\lambda^{2} s-\lambda h\right|^{\theta-1}(1+\sqrt{\lambda}|\lambda s-h|)^{-N}+e^{-c h^{2} \lambda}\right) d h \leq \lambda^{-\frac{\theta}{2}}\left|\lambda^{2} s\right|^{\theta-1}
$$

for

$$
1 \leq \theta \leq \frac{n+1}{2}, \quad|s| \gtrsim \lambda^{-\frac{3}{2}}
$$

This is again straightforward. 
It remains to prove the kernel bound (54). The integrand involves the Hamilton flow $(x, \xi) \rightarrow\left(x_{h}, \xi_{h}\right)$. Hence, our next goal is to study the regularity of the flow map.

4.10. The regularity of the Hamilton flow. Here we obtain precise bounds on the derivatives of the flow map $F^{h}$ with respect to $\xi$. Observe first that if the $\left(C^{2}\right)$ coefficients $a^{i j}$ have Fourier transform supported in $|\xi| \leq \sqrt{\lambda}$ then the following relations hold:

$$
\left|\partial_{x}^{\alpha} a^{i j}\right| \leq c_{\alpha} \lambda^{\frac{\alpha-2}{2}},|\alpha| \geq 2
$$

We start with a weaker result which we strengthen afterwards:

Lemma 6. Assume that the coefficients of $P$ are of class $C^{2}$, with Fourier transform supported in $B(0, \sqrt{\lambda})$. Then the following bounds hold

$$
\begin{aligned}
& \left|\partial_{\xi}^{\alpha} x_{h}\right| \leq h(1+h \sqrt{\lambda})^{|\alpha|-1}, \quad|\alpha| \geq 1 \\
& \left|\partial_{\xi}^{\alpha} \xi_{h}\right| \leq(1+h \sqrt{\lambda})^{|\alpha|-1} \quad|\alpha| \geq 1
\end{aligned}
$$

Proof. We use induction with respect to $\alpha$. For $\alpha=1$ the functions $\left(\partial_{\xi} x_{h}, \partial_{\xi} \xi_{h}\right)$ solve the linearized system

$$
\frac{d}{d h}\left(\begin{array}{c}
y \\
\eta
\end{array}\right)=A(h)\left(\begin{array}{c}
y \\
\eta
\end{array}\right), \quad A(h)=\left(\begin{array}{cc}
a_{\xi x}\left(x_{h}, \xi_{h}\right) & a_{\xi \xi}\left(x_{h}, \xi_{h}\right) \\
-a_{x x}\left(x_{h}, \xi_{h}\right) & -a_{x \xi}\left(x_{h}, \xi_{h}\right)
\end{array}\right)
$$

with initial data

$$
\left(\begin{array}{c}
y(0) \\
\eta(0)
\end{array}\right)=\left(\begin{array}{c}
0 \\
I_{n}
\end{array}\right)
$$

Since $A(h)$ is bounded, it follows that the solutions remain bounded. Furthermore, the Cauchy data of the first component is 0 , therefore the first component can be bounded by $c h$.

For the induction step, compute

$$
\frac{d}{d h} \partial_{\xi}^{\alpha} x_{h}=\partial_{\xi}^{\alpha} \frac{d}{d h} x_{h}=\partial_{\xi}^{\alpha} a_{\xi}\left(x_{h}, \xi_{h}\right)
$$

and

$$
\frac{d}{d h} \partial_{\xi}^{\alpha} \xi_{h}=\partial_{\xi}^{\alpha} \frac{d}{d h} \xi_{h}=-\partial_{\xi}^{\alpha} a_{\xi}\left(x_{h}, \xi_{h}\right)
$$

Then the functions $\left(\frac{d}{d t} \partial_{\xi}^{\alpha} x_{t}, \frac{d}{d t} \partial_{\xi}^{\alpha} \xi_{t}\right)$ solve a system of the form

$$
\frac{d}{d h}\left(\begin{array}{l}
y \\
\eta
\end{array}\right)=A(h)\left(\begin{array}{l}
y \\
\eta
\end{array}\right)+\left(\begin{array}{l}
\bar{y}(h) \\
\bar{\eta}(h)
\end{array}\right)
$$

with zero initial data, where the inhomogeneous term $(\bar{y}(h), \bar{\eta}(h))$ can be expressed in terms of the lower order derivatives as a sum of products of the form

$$
\left(\partial_{x}^{\alpha_{1}} \partial_{\xi}^{\alpha_{2}} a\right)\left(x_{h}, \xi_{h}\right) \prod_{k=1}^{\left|\alpha_{1}\right|}\left(\partial^{\alpha_{1}^{k}} x_{h}\right) \prod_{k=1}^{\left|\alpha_{2}\right|}\left(\partial_{\xi}^{\alpha_{2}^{k}} \xi_{h}\right)
$$

where

$$
1 \leq\left|\alpha_{1}\right|+\left|\alpha_{2}\right| \leq|\alpha|, \quad 1 \leq\left|\alpha_{1}^{k}\right|,\left|\alpha_{2}^{k}\right| \leq|\alpha|-1 \quad \sum\left|\alpha_{1}^{k}\right|+\left|\alpha_{2}\right|^{k}=|\alpha|
$$

Since the coefficients of $P$ are of class $C^{2}$, with Fourier transform supported in $B(0, \sqrt{\lambda})$, it follows that

$$
\left(\partial_{x}^{\alpha_{1}} \partial_{\xi}^{\alpha_{2}} a\right)\left(x_{h}, \xi_{h}\right) \leq c_{\alpha_{1}, \alpha_{2}} \lambda^{\frac{\left|\alpha_{1}\right|-2}{2}}, \quad\left|\alpha_{1}\right| \geq 2
$$


Then, given the induction hypothesis, we can bound both $\bar{y}(h)$ and $\bar{\eta}(h)$ by $(1+$ $t \sqrt{\lambda})^{|\alpha|-1}$ and the desired bound follows by integrating the system.

We can use the above Lemma to produce an expansion of $x_{h}, \xi_{h}$ in terms of powers of $h$ :

Lemma 7. Assume that the coefficients of $P$ are of class $C^{2}$, with Fourier transform supported in $B(0, \sqrt{\lambda})$. Then the following estimates hold:

$$
\begin{gathered}
x_{h}=x+h a_{\xi}+h^{2} g(h, x, \xi) \\
\xi_{h}=\xi+h m(h, x, \xi)
\end{gathered}
$$

where $g, m$ satisfy the following bounds:

$$
\left|\partial_{\xi}^{\alpha} m(h, x, \xi)\right|,\left|\partial_{\xi}^{\alpha} g(h, x, \xi)\right| \leq(1+h \sqrt{\lambda})^{|\alpha|-1},
$$

Proof. We have

$$
\begin{aligned}
x_{h} & =x+h a_{\xi}+\int_{0}^{h}(h-\tilde{h}) \frac{d^{2}}{d \tilde{h}^{2}} x_{\tilde{h}} d \tilde{h} \\
& =x+h a_{\xi}+\int_{0}^{h}(h-\tilde{h}) f\left(x_{\tilde{h}}, \xi_{\tilde{h}}\right) d \tilde{h}
\end{aligned}
$$

where $f$ can be computed from the flow equation,

$$
f(x, \xi)=a_{\xi x} a_{\xi}-a_{\xi \xi} a_{x}
$$

Then it suffices to estimate the derivatives

$$
\left|\partial_{\xi}^{\alpha} f\left(x_{h}, \xi_{h}\right)\right| \leq 1+h(h \sqrt{\lambda})^{|\alpha|-1},
$$

By hypothesis

$$
\left|\partial_{x}^{\alpha} \partial_{\xi}^{\beta} f(x, \xi)\right| \leq c_{\alpha, \beta}(\sqrt{\lambda})^{|\alpha|-1}
$$

so this can be easily done using the previous Lemma. The estimate for $\xi_{h}$ is similar.

Another straightforward consequence of Lemma 6 is the following bound for the Gaussians in our kernel:

Lemma 8. For $\xi$ in a compact set and away from 0 we have

$$
\left|\partial_{\xi}^{\alpha} G\left(t, x_{h}, \xi_{h}\right)\right| \leq c_{\alpha} e^{-c \lambda\left(x_{h}-\tilde{y}\right)^{2}}(1+h \sqrt{\lambda})^{\alpha},
$$

It is somewhat more difficult to prove a related result for the exponent in the "bad" oscillatory term in our kernel,

$$
\phi_{h}(t, x, \xi)=\lambda\left[\left(\tilde{y}-x_{h}\right) \xi_{h}+2 h a(t, x, \xi)\right]
$$

Choose $\tilde{\xi}$ so that $\tilde{y}=\tilde{x}_{h}$. By $(60)$ we have

$$
\left|x_{h}-\tilde{x}_{h}\right| \approx h|\xi-\tilde{\xi}|
$$

Hence it follows that

$$
(x-y)^{2}+h^{2}(\xi-\tilde{\xi})^{2} \leq c\left[(x-y)^{2}+\left(x_{h}-\tilde{y}\right)^{2}\right]
$$

Now we can state our result 
Lemma 9. For $\xi$ in a compact set and away from 0 we have

$$
\left|\partial_{\xi}\left(\phi_{h}(t, x, \xi)+h \lambda \xi a_{\xi}(t, x, \tilde{\xi})\right)\right| \leq\left(1+\lambda\left(x_{h}-\tilde{y}\right)^{2}\right)(1+h \sqrt{\lambda})
$$

and

$$
\left|\partial_{\xi}^{\alpha} \phi_{h}(t, x, \xi)\right| \leq c_{\alpha}\left(1+\lambda\left(x_{h}-\tilde{y}\right)^{2}\right)(1+h \sqrt{\lambda})^{\alpha},|\alpha| \geq 2
$$

In the case when $|h| \leq \lambda^{-\frac{1}{2}}$, we can replace (64) with

$$
\left|\partial_{\xi}\left(\phi_{h}(t, x, \xi)+\lambda(\tilde{y}-x) \xi\right)\right| \leq\left(1+\lambda\left(x_{h}-\tilde{y}\right)^{2}\right)(1+h \sqrt{\lambda})
$$

The above bound on the second and higher derivatives of the phase shows that we can replace the phase by a linear one modulo a factor which can be included in the Gaussians. This will allow us later to work with phase functions which are linear in $\xi$.

Proof. Observe that

$$
\xi_{h} \cdot \partial_{\xi} x_{h}=h a_{\xi}(t, x, \xi)
$$

Indeed, this is trivially true at $h=0$. If we use the linearized equations (59) then we get

$$
\frac{d}{d h}\left(\xi_{h} \cdot \partial_{\xi} x_{h}\right)=-a_{x} \cdot \partial_{\xi} x_{h}+\xi_{h} \cdot\left(a_{\xi x} \partial_{\xi} x_{h}+a_{\xi \xi} \partial_{\xi} \xi_{h}\right)
$$

Since $a$ is homogeneous of order 2 in $\xi$, this implies that

$$
\frac{d}{d h}\left(\xi_{h} \cdot \partial_{\xi} x_{h}\right)=\left(a_{x} \partial_{\xi} x_{h}+a_{\xi} \partial_{\xi} \xi_{h}\right)=\partial_{\xi} a\left(t, x_{h}, \xi_{h}\right)=\partial_{\xi} a(t, x, \xi)
$$

Hence (67) follows.

Then the first derivative of $\phi_{h}$ is

$$
\begin{aligned}
\partial_{\xi} \phi_{h}(t, x, \xi) & =\lambda\left(\left(\tilde{y}-x_{h}\right) \partial_{\xi} \xi_{h}+2 h a_{\xi}(t, x, \xi)\right) \\
& =h \lambda\left(\partial_{\xi} m(t, x, \xi)\right)\left(\tilde{y}-x_{h}\right)+\lambda\left(\tilde{y}-x_{h}\right)+h \lambda a_{\xi}(t, x, \xi) \\
& \left.=h \lambda\left(\partial_{\xi} m(t, x, \xi)\right)\left(\tilde{y}-x_{h}\right)+\lambda(\tilde{y}-x)-h^{2} \lambda g(t, x, \xi)\right)
\end{aligned}
$$

Now (65) follows easily from the bounds on the derivatives of $x_{h}$ in Lemma 6 and the bounds on the derivatives of $g, m$ in Lemma 7 . To prove (64) it suffices to observe that the $\xi$ gradient of $\phi_{h}(t, x, \xi)+h \lambda \xi a_{\xi}(t, x, \tilde{\xi})$ vanishes at $\xi=\tilde{\xi}$. Then we can use (65) with $|\alpha|=2$ to obtain

$$
\left|\partial_{\xi}\left(\phi_{h}(t, x, \xi)+h \lambda \xi a_{\xi}(t, x, \tilde{\xi})\right)\right| \lesssim\left(1+\lambda\left(x_{h}-\tilde{y}\right)^{2}\right)(1+h \sqrt{\lambda})^{2}|\xi-\tilde{\xi}|
$$

Thus (64) follows by (63).

4.11. The short range kernel estimate. We want to estimate the kernel

$$
\begin{aligned}
H^{h}(\tilde{s}, \tilde{y}, s, y)= & \lambda^{\frac{3(n+2)}{2}} \int_{K} G(t, \xi, x, y) G\left(t, \xi_{h}, x_{h}, \tilde{y}\right) e^{-\frac{\lambda^{2}}{2}(t-s)^{2}} \\
& e^{-\frac{\lambda^{2}}{2}(t-\tilde{s})^{2}} e^{i \lambda^{2} \tau(\tilde{s}-s)} e^{i \lambda \xi(x-y)} e^{i \lambda \xi_{h}\left(\tilde{y}-x_{h}\right)} e^{2 i \lambda \tau h} d t d x d \tau d \xi
\end{aligned}
$$

in (54) for $h<\lambda^{-\frac{1}{2}}$. Due to Lemmas 8,9 and the gaussian bounds (41) on $G$, the integrand can be represented in the form

$$
e^{-c \lambda(x-y)^{2}} e^{-c \lambda(x-\tilde{y})^{2}} e^{-\frac{\lambda^{2}}{2}(t-s)^{2}} e^{-\frac{\lambda^{2}}{2}(t-\tilde{s})^{2}} e^{i \lambda^{2} \tau(\tilde{s}-s)} e^{i \lambda \xi(y-\tilde{y})} f(t, y, \tilde{y}, x, \xi)
$$


where $f$ is bounded, compactly supported away from $\xi=0$ and has bounded derivatives in $\xi$. Hence the kernel $H^{h}$ can be represented as

$$
\begin{aligned}
H^{h}(\tilde{s}, \tilde{y}, s, y)= & \lambda^{\frac{3(n+2)}{2}} \int_{K} e^{-c \lambda(x-y)^{2}} e^{-c \lambda(x-\tilde{y})^{2}} e^{i \lambda^{2} \tau(\tilde{s}-s)} e^{i \lambda \xi(\tilde{y}-y)} \\
& e^{-\frac{\lambda^{2}}{2}(t-s)^{2}} e^{-\frac{\lambda^{2}}{2}(t-\tilde{s})^{2}} f(t, y, \tilde{y}, x, \xi) d t d x d \tau d \xi
\end{aligned}
$$

To estimate this we use standard oscillatory integral estimates of the form

$$
\int_{K} f(\xi, \tau) e^{i(y \xi+\tau s)} d \xi d \tau \lesssim(1+|y|+|s|)^{-\frac{n}{2}}\left(\frac{1+|y|}{1+|y|+|s|}\right)^{N}
$$

for $f$ smooth, compactly supported, vanishing near 0 . What is important in these oscillatory integral estimates is that the characteristic set $K=\{p=0\}$ has $n$ nonvanishing curvatures (see e.g. Stein [17] 8.3.1 and 9.1.2). The decay given by the first factor on the right is sharp in the cone of directions in the conormal bundle of $K \cap \operatorname{supp} f$. Away from this cone, the integral is rapidly decreasing. Since $f$ is supported away from 0 it follows that our oscillatory integral is rapidly decreasing in a conical neighborhood of $y=0$. This motivates the second factor in the right hand side of (68).

From (68) we obtain

$$
\begin{aligned}
\left|H^{h}(s, \tilde{s}, y, \tilde{y})\right| \leq & \lambda^{\frac{3(n+2)}{2}}\left(1+\lambda^{2}|s-\tilde{s}|\right)^{-\frac{n}{2}}\left(\frac{1+\lambda|y-\tilde{y}|}{1+\lambda|y-\tilde{y}|+\lambda^{2}|s-\tilde{s}|}\right)^{N} \\
& \int e^{-\frac{\lambda^{2}}{2}(t-s)^{2}} e^{-\frac{\lambda^{2}}{2}(t-\tilde{s})^{2}} e^{-c \lambda(x-y)^{2}} e^{-c \lambda(x-\tilde{y})^{2}} d t d x
\end{aligned}
$$

Then the desired bound (54) follows after integration in $x, t$.

4.12. The long range estimate. As in the short range estimate, our strategy will be to replace the phase function by a linear one and treat everything else as a perturbation. This time the derivatives in $\xi$ are of the order of $(h \sqrt{\lambda})^{|\alpha|}$, but this is compensated by the fact that our estimates are localized in $\xi$ on the scale of $(h \sqrt{\lambda})^{-1}$.

Again, due to Lemmas 8,9 and the gaussian bounds (41) on $G$, the integrand can be represented in the form

$$
\begin{aligned}
E_{h}(s, y, \tilde{s}, \tilde{y}, t, x, \tau, \xi)= & e^{-c \lambda(x-y)^{2}} e^{-c \lambda h^{2}(\xi-\tilde{\xi})^{2}} e^{-\frac{\lambda^{2}}{2}(t-s)^{2}} e^{-\frac{\lambda^{2}}{2}(t-\tilde{s})^{2}} \\
& e^{i \lambda^{2} \tau(s-\tilde{s})} e^{i h \lambda \xi p_{\xi}(t, x, \tilde{\xi})} f(t, y, \tilde{y}, x, \xi)
\end{aligned}
$$

where $f$ satisfies

$$
\left|\partial_{\xi}^{\alpha} f\right| \leq c_{\alpha}(1+h \sqrt{\lambda})^{\alpha}
$$

Here $\tilde{\xi}$ depends on $t, x, y, \tilde{y}$ but this does not matter since we estimate the $\xi$ integral first. We need to prove that

$$
\lambda^{\frac{3(n+2)}{2}}\left|\int_{K} E_{h} d t d x d \xi\right| \leq c \lambda^{n+2}\left(1+\lambda^{2}|s-\tilde{s}|\right)^{-\frac{n}{2}}\left((\sqrt{\lambda}(\lambda(s-\tilde{s})-h))^{-N}+e^{-c \lambda h^{2}}\right)
$$


This follows after a straightforward integration in $x, t$ from the corresponding bound for the integral with respect to $\xi$,

$$
\begin{array}{r}
\left|\int_{K} e^{i \lambda^{2} \tau(s-\tilde{s})} e^{i h \lambda \xi p_{\xi}(t, x, \tilde{\xi})} e^{-c \lambda h^{2}(\xi-\tilde{\xi})^{2}} f(\xi) d \xi\right| \lesssim \\
\left(1+\lambda^{2}|s-\tilde{s}|\right)^{-\frac{n}{2}}\left((\sqrt{\lambda}(\lambda(s-\tilde{s})-h))^{-N}+e^{-c h^{2} \lambda}\right)
\end{array}
$$

for $f$ compactly supported away from 0 and satisfying (69). In the region $|\xi-\tilde{\xi}| \gtrsim 1$ we have

$$
\left|\partial_{\xi}^{\alpha} e^{-c \lambda h^{2}(\xi-\tilde{\xi})^{2}} f(\xi)\right| \leq c_{\alpha} e^{-c_{1} \lambda h^{2}}
$$

therefore the stationary phase estimate (68) yields a bound of $e^{-c_{1} h^{2} \lambda}\left(1+\lambda^{2} \mid s-\right.$ $\tilde{s} \mid)^{-\frac{n}{2}}$ for the integral.

It remains to consider the case when $f$ is supported in a small neighborhood of $\tilde{\xi}$. In this case we must necessarily have $|\tilde{\xi}|=O(1)$. The argument simplifies considerably if we take into account the fact that $a$ is quadratic in $\xi$. Then we can make the substitution

$$
s:=s-\tilde{s}, \quad \xi:=\xi-\tilde{\xi}
$$

The new phase function is

$$
\lambda^{2} a(\xi+\tilde{\xi}) s-h \lambda(\xi+\tilde{\xi}) p_{\xi}(\tilde{\xi})=\lambda^{2} a(\xi) s+\lambda(\lambda s-h) \xi p_{\xi}(\tilde{\xi})+\lambda^{2} a(\tilde{\xi}) s
$$

and the estimate to prove is

$$
\left|\int_{K} e^{i \lambda^{2} \tau s} e^{i \lambda(\lambda s-h) \xi a_{\xi}(t, x, \tilde{\xi})} e^{-c \lambda h^{2} \xi^{2}} f(\xi) d \xi\right| \lesssim\left(1+\lambda^{2} s\right)^{-\frac{n}{2}}(\sqrt{\lambda}(\lambda s-h))^{-N}
$$

with $f$ supported in a small neighborhood of 0 .

If $|\lambda s| \lesssim|\lambda s-h|$ then the phase is nondegenerate, and a simple integration by parts yields

$$
\left|\int_{K} e^{i \lambda^{2} \tau s} e^{i \lambda(\lambda s-h) \xi p_{\xi}(t, x, \tilde{\xi})} e^{-c \lambda h^{2} \xi^{2}} f(\xi) d \xi\right| \lesssim(h \sqrt{\lambda})^{-\frac{n}{2}}\left(\frac{h \sqrt{\lambda}}{\left(1+\lambda^{2}|s|+\lambda|h|\right)}\right)^{N}
$$

which is more than we need.

Finally, we consider the interesting case, i.e. when $|\lambda s-h| \ll|\lambda s|$. Then $\lambda s=O(h)$. After a linear change of coordinates we can assume that $a(\xi)=\xi^{2}$. Set $t=\lambda s, x=(\lambda s-h) a_{\xi}(\tilde{\xi})$.

Then we have to show that

$$
\left|\int e^{i \lambda t \xi^{2}} e^{i \lambda x \xi} e^{-\lambda t^{2} \xi^{2}} f(\xi) d \xi\right| \lesssim(1+\lambda|t|)^{-\frac{n}{2}}(1+\sqrt{\lambda}|x|)^{-N}, \quad|x| \lesssim|t| \lesssim 1
$$

where

$$
\left|\partial^{\alpha} f\right| \leq c_{\alpha}(\sqrt{\lambda}|t|)^{\alpha}
$$

We interpret the last integral as a convolution of the Fourier transforms of $e^{i \lambda t \xi^{2}} e^{-\lambda t^{2} \xi^{2}}$, respectively $e^{-\lambda t^{2} \xi^{2}} f(\xi)$. We have

$$
\begin{aligned}
\left|\int e^{i x \xi} e^{i \lambda t \xi^{2}} e^{-\lambda t^{2} \xi^{2}} d \xi\right| & =c\left|\left(i \lambda t+\lambda t^{2}\right)^{-\frac{n}{2}} e^{\frac{x^{2}}{-\lambda t^{2}+i \lambda t}}\right| \\
& \lesssim(\lambda t)^{-\frac{n}{2}} e^{-c \frac{x^{2}}{\lambda}}
\end{aligned}
$$

while

$$
\left|\int e^{i x \xi} e^{-\lambda t^{2} \xi^{2}} f(\xi) d \xi\right| \lesssim(t \sqrt{\lambda})^{-n}\left(1+\frac{|x|}{t \sqrt{\lambda}}\right)^{-N}
$$


Then their convolution is bounded by

$$
\left|(\lambda t)^{-\frac{n}{2}} e^{-c \frac{x^{2}}{\lambda}} \star(t \sqrt{\lambda})^{-n}\left(1+\frac{|x|}{t \sqrt{\lambda}}\right)^{-N}\right| \lesssim(\lambda t)^{-\frac{n}{2}}\left(1+\frac{|x|}{\sqrt{\lambda}}\right)^{-N}
$$

Replacing $x$ by $\lambda x$ we get (70).

\section{REFERENCES}

[1] Hajer Bahouri and Jean-Yves Chemin. Équations d'ondes quasilinéaires et effet dispersif. Internat. Math. Res. Notices, 1999(21):1141-1178.

[2] Hajer Bahouri and Jean-Yves Chemin. Équations d'ondes quasilinéaires et estimations de Strichartz. Amer. J. Math., 121(6):1337-1377, 1999.

[3] W. Craig, T. Kappler, and W. Strauss. Microlocal dispersive smoothing for the Schrödinger equation. Comm. Pure Appl. Math., 48(8):769-786, 1995.

[4] Shin-ichi Doi. On the Cauchy problem for Schrödinger type equations and the regularity of solutions. J. Math. Kyoto Univ., 34(2):319-328, 1994.

[5] Shin-ichi Doi. Remarks on the Cauchy problem for Schrödinger-type equations. Comm. Partial Differential Equations, 21(1-2):163-178, 1996.

[6] J. Ginibre. An introduction to nonlinear Schrödinger equations. In Nonlinear waves (Sapporo, 1995), pages 85-133. Gakkōtosho, Tokyo, 1997.

[7] Lars Hörmander. The analysis of linear partial differential operators. I-IV. Springer-Verlag, Berlin, 1985.

[8] L. Kapitanski and Y. Safarov. Dispersive smoothing for Schrödinger equations. Math. Res. Letters, 3:77-91, 1996.

[9] L. Kapitanski and Yu. Safarov. A parametrix for the nonstationary Schrödinger equation. In Differential operators and spectral theory, pages 139-148. Amer. Math. Soc., Providence, RI, 1999.

[10] Markus Keel and Terence Tao. Endpoint Strichartz estimates. Amer. J. Math., 120(5):955980, 1998.

[11] Carlos E. Kenig, Gustavo Ponce, and Luis Vega. personal communication.

[12] Carlos E. Kenig, Gustavo Ponce, and Luis Vega. Small solutions to nonlinear Schrödinger equations. Ann. Inst. H. Poincaré Anal. Non Linéaire, 10(3):255-288, 1993.

[13] S. Klainerman and I Rodnianski. personal communication.

[14] C. Rolvung. Nonisotropic Schrödinger equations. 1998. Ph. D. Thesis, Department of Mathematics, University of Chicago.

[15] Hart F. Smith. A parametrix construction for wave equations with $C^{1,1}$ coefficients. Ann. Inst. Fourier (Grenoble), 48(3):797-835, 1998.

[16] Hart F. Smith and Christopher Sogge. Global strichartz estimates for nontrapping perturbations of the laplacian. to appear, Comm. PDE.

[17] Elias M. Stein. Harmonic analysis: real-variable methods, orthogonality, and oscillatory integrals. Princeton University Press, Princeton, NJ, 1993. With the assistance of Timothy S. Murphy, Monographs in Harmonic Analysis, III.

[18] Daniel Tataru. Strichartz estimates for operators with nonsmooth coefficients iii. to appear, AMS Journal.

[19] Daniel Tataru. Strichartz estimates for operators with nonsmooth coefficients and the nonlinear wave equation. Amer. J. Math., 122(2):349-376, 2000.

[20] Daniel Tataru. Strichartz estimates for second order hyperbolic operators with nonsmooth coefficients. II. Amer. J. Math., 123(3):385-423, 2001.

[21] Michael E. Taylor. Partial differential equations. III. Springer-Verlag, New York, 1997. Nonlinear equations, Corrected reprint of the 1996 original.

Department of Mathematics, Stanford University

Department of Mathematics, Northwestern University 